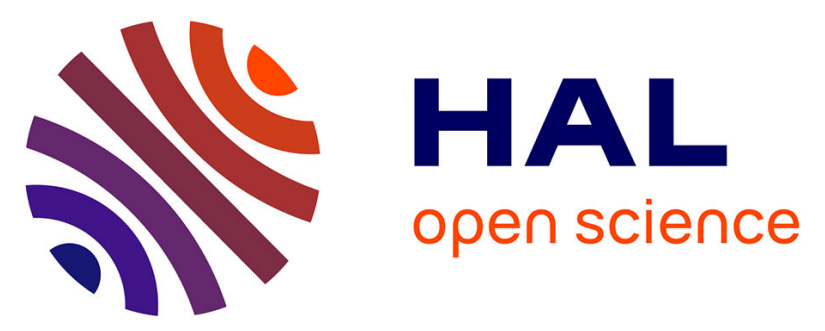

\title{
Subpercent agreement between ab initio and experimental collision-induced line shapes of carbon monoxide perturbed by argon
}

Grzegorz Kowzan, Hubert Cybulski, Piotr Wcislo, Michal Slowiński, Alexandra Viel, Piotr Maslowski, Franck Thibault

\section{To cite this version:}

Grzegorz Kowzan, Hubert Cybulski, Piotr Wcislo, Michal Slowiński, Alexandra Viel, et al.. Subpercent agreement between ab initio and experimental collision-induced line shapes of carbon monoxide perturbed by argon. Physical Review A, 2020, 102 (1), pp.012821. 10.1103/PhysRevA.102.012821. hal-02908662

\section{HAL Id: hal-02908662 \\ https://hal.science/hal-02908662}

Submitted on 29 Jul 2020

HAL is a multi-disciplinary open access archive for the deposit and dissemination of scientific research documents, whether they are published or not. The documents may come from teaching and research institutions in France or abroad, or from public or private research centers.
L'archive ouverte pluridisciplinaire HAL, est destinée au dépôt et à la diffusion de documents scientifiques de niveau recherche, publiés ou non, émanant des établissements d'enseignement et de recherche français ou étrangers, des laboratoires publics ou privés. 


\title{
Subpercent agreement between $a b$ initio and experimental collision-induced line shapes of carbon monoxide perturbed by argon
}

\author{
Grzegorz Kowzan $\odot,{ }^{1, *}$ Hubert Cybulski $\odot,{ }^{2}$ Piotr Wcisło, ${ }^{1}$ Michał Słowiński $\odot,{ }^{1}$ Alexandra Viel, ${ }^{3}$ \\ Piotr Masłowski, ${ }^{1}$ and Franck Thibault (i) $^{3}$ \\ ${ }^{1}$ Institute of Physics, Faculty of Physics, Astronomy and Informatics, Nicolaus Copernicus University in Toruń, ul. \\ Grudziadzka 5, 87-100 Toruń, Poland \\ ${ }^{2}$ Institute of Physics, Kazimierz. Wielki University, ul. Powstańców Wielkopolskich 2, 85-090 Bydgoszcz, Poland \\ ${ }^{3}$ Univ Rennes, CNRS, IPR (Institut de Physique de Rennes)-UMR 6251, F-35000 Rennes, France
}

(Received 31 May 2020; accepted 10 July 2020; published 28 July 2020)

\begin{abstract}
We present fully $a b$ initio calculations of second-overtone rovibrational line shapes of carbon monoxide perturbed by argon. The quantum mechanical scattering problem between $\mathrm{CO}$ and Ar is solved numerically for two different $a b$ initio interaction potentials. We use the generalized Hess method to determine spectroscopic cross sections which describe the effect of collisions on each spectral line. Using these cross sections, we determine the line-shape parameters that we use to generate the Hartmann-Tran and speed-dependent billiard ball profiles. We compare the generated line shapes with high-quality experimental line profiles of five lines measured at five pressures between 0.01 and $1 \mathrm{~atm}$. A subpercent agreement over the entire pressure range is obtained. Calculations for the $\mathrm{P}(9)$ line are used to inspect the effects of the two interaction potentials. The discrepancies for both the considered interaction potentials and the experiment are explained within the described theoretical framework. The presented results are the most accurate collisional line-shape calculations for a system with collision dynamics representative of atmospherically relevant species.
\end{abstract}

DOI: 10.1103/PhysRevA.102.012821

\section{INTRODUCTION}

The ability to accurately predict and interpret molecular spectra is important for many purposes. Studies of the atmosphere of Earth [1-3] and other planets [4] depend on the availability of spectroscopic reference data [5], which are obtained from laboratory measurements [6] and supplemented by classical molecular dynamics simulations (CMDSs) $[7,8]$. The accuracy of molecular interaction potentials can be assessed by comparison with simulated spectral line shapes [9]. Establishing an accurate link between the thermodynamic temperature and the Doppler line widths hinges on a proper interpretation of the full line shape [10]. For molecular hydrogen, confronting the experimental transition frequencies with theoretical predictions [11] allows testing of the limits of validity of quantum electrodynamics for molecules or searching for new physics beyond the Standard Model [12]. In all these cases, it is crucial to properly account for the collisional effects in molecular spectra [13], including the speed dependence of line broadening and shift [14] and the influence of collision-induced velocity changes [15].

A fully $a b$ initio method of determining molecular line shapes is desirable because it allows us to verify our understanding of the line-shape problem and generate reference line-shape parameters without time-consuming, expensive, and technically challenging experiments. The speeddependent billiard ball profile (SDBBP) [16] is based on a

\footnotetext{
*grzegorz@kowzan.eu
}

numerical solution of a quantum Boltzmann equation and it uses a physically justified model of collision-induced velocity changes (VCs), in contrast to commonly used phenomenological profiles such as the Hartmann-Tran profile (HTP) [17]. For this reason, the SDBBP has been successful in explaining the collisional inhomogenous broadening in $\mathrm{H}_{2}-\mathrm{Ar}$ spectra [18], determining transition frequencies in $\mathrm{D}_{2}$ with a high accuracy [19], and modeling Dicke-narrowed spectra of COAr [20,21].

For a complete description of the line shape, a procedure for calculating the parameters describing the influence of collisions on internal and external molecular degrees of freedom is additionally needed. We have recently described this procedure [22,23], limited to the case of an isolated line within binary collision impact theory [24]. Within these restrictions, collisions can change the phase and state of the active molecule, producing broadening and shift of the line. (In the following, the change of phase or state is called dephasing for short.) Collisions can also change the velocity of the molecule, hindering its free motion responsible for the Doppler width, and narrow the profile [15]. Pressure broadening is customarily separated into its thermally averaged part and its speeddependent part, with the latter narrowing the line profile. In the case of pressure shift, its speed dependence may broaden the line and also make it asymmetric. Asymmetry of the line also occurs because dephasing and velocity-changing effects cannot be rigorously separated [25]. Quantum-mechanical calculations of collisional dephasing have been performed for numerous systems [13,26], including CO-Ar [20,21,27,28]. In contrast, treatments accounting for both dephasing and the 
effects of VCs were mostly limited to the hydrogen molecule and its isotopologues [19,22,29-31], which are not representative of systems relevant to atmospheric studies.

In this work, we present fully $a b$ initio collisional line shapes of CO-Ar rovibrational transitions in the $\mathrm{P}$ branch of the second-overtone band. The collisional line-shape parameters describing both dephasing and velocity-changing effects were obtained from the solution of the quantum-mechanical scattering problem for an $a b$ initio potential. We directly compare the generated line profiles with high-SNR (signalto-noise ratio) experimental spectra [32], acquired with a comb-assisted cavity ringdown spectroscopy system [33-35]. The difference between $a b$ initio and experimental spectra is subpercent over the whole pressure range-from the strongly Dicke-narrowed regime to the pressure-broadening regime. We emphasize that no collisional line-shape parameter was adjusted to the experiment. This is the first time this level of agreement was reached for a system with collision dynamics typical of atmospherically relevant species. Moreover, we find that using the recently described procedure for approximating the SDBBP with the HTP [36] results in similarly small discrepancies, paving the way for $a b$ initio generation of spectroscopic reference data. We use these results to identify the source of discrepancies in $a b$ initio line-shape parameters based on two different potential energy surfaces versus those derived from experimental line shapes.

The paper is organized as follows: The theoretical framework is described in Sec. II. The results are presented in Sec. III, which contains the computational details (Sec. III A), a direct comparison between generated and measured line shapes (Sec. III B), and a comparison between calculated and fitted line-shape parameters (Sec. III C). Finally, the article is concluded in Sec. IV. A comparison between potential energy surfaces is given in Appendix B.

\section{LINE-SHAPE PROBLEM}

The formal solution of the line-shape problem is given by the integral

$$
I(\omega)=\frac{1}{\pi} \operatorname{Re} \int_{\mathbb{R}^{3}} \mathrm{~d} \vec{v}_{1} \rho_{b a}\left(\omega, \vec{v}_{1}\right),
$$

where $\omega$ is the angular frequency of incident radiation, $\vec{v}_{1}$ is the absorber speed, and $\rho_{b a}\left(\omega, \vec{v}_{1}\right)$ is the velocity distribution of the optical coherence between the initial and final state, $a, b$, of the absorber. The rotational (vibrational) numbers corresponding to $a, b$ states are denoted $j_{a}, j_{b}\left(v_{a}, v_{b}\right)$. The full density matrix, $\rho\left(\omega, \vec{v}_{1}\right)$, includes multiple degenerate states associated with each rotational state, $|v j m\rangle$, so the $\rho_{b a}\left(\omega, \vec{v}_{1}\right)$ distribution is obtained by averaging over all $m_{a}, m_{b}$ states associated with $j_{a}, j_{b}$ quantum numbers. Assuming no collisional coupling between coherences, i.e., isolated lines, the averaging is given by [37]

$$
\rho_{b a}\left(\omega, \vec{v}_{1}\right)=\operatorname{Tr}\left[T_{b a}^{(q) n^{\dagger}} \rho\left(\omega, \vec{v}_{1}\right)\right]
$$

where $\operatorname{Tr}$ is the trace over internal states of the molecule. In the above equation, the $T_{b a}^{(q) n}$ spherical tensor operator is a state multipole given by $[37,38]$

$$
\begin{aligned}
T_{b a}^{(q) n}= & i^{q}(2 q+1)^{1 / 2} \sum_{m_{b}, m_{a}}(-1)^{j_{b}-m_{b}}\left(\begin{array}{ccc}
j_{b} & q & j_{a} \\
-m_{b} & n & m_{a}
\end{array}\right) \\
& \times\left|v_{b} j_{b} m_{b}\right\rangle\left\langle v_{a} j_{a} m_{a}\right|,
\end{aligned}
$$

where $(:::)$ is the $3-j$ symbol, $q$ is the tensor order of the considered transition, and $n$ is its projection. The $\rho_{b a}\left(\omega, \vec{v}_{1}\right)$ distribution can be obtained from the generalized WaldmannSnider equation $[39,40]$,

$$
1=\left[-i\left(\omega-\omega_{0}-\vec{k} \cdot \vec{v}_{1}\right)-\hat{S}_{b a}\right] \tilde{\rho}_{b a}\left(\omega, \vec{v}_{1}\right),
$$

where $\tilde{\rho}_{b a}\left(\omega, \vec{v}_{1}\right)$ is the optical coherence distribution with the Maxwell-Boltzmann distribution factored out, $\rho_{b a}\left(\omega, \vec{v}_{1}\right)=$ $\tilde{\rho}_{b a}\left(\omega, \vec{v}_{1}\right) f_{\mathrm{MB}}\left(\vec{v}_{1}\right), \vec{k} \cdot \vec{v}_{1}$ describes the Doppler shift, and $\omega_{0}$ is the angular frequency of the transition.

In the SDBBP the collision operator $\hat{S}_{b a}$ is assumed to adopt the form

$$
\hat{S}_{b a}=-\Gamma\left(v_{1}\right)-i \Delta\left(v_{1}\right)+v_{\mathrm{opt}} \hat{M}_{\mathrm{BB}},
$$

where $\Gamma\left(v_{1}\right)$ and $\Delta\left(v_{1}\right)$ are the speed-dependent broadening and shift, $v_{\mathrm{opt}}$ is the complex Dicke parameter, and $\hat{M}_{\mathrm{BB}}$ is the normalized billiard ball operator [19]. The real part of the complex Dicke parameter is smaller [41,42] than the frequency of VCs associated with mass diffusion, $v_{\text {diff. This }}$ discrepancy has been partially explained by the dependence of the specific $v_{\mathrm{opt}}$ value on the model of VCs in collisions $[36,43]$. The frequency of VCs is additionally reduced by discounting the collisions that both remove the coherence between radiative states and change the velocity, since they cannot affect the line shape. Equation (5) with $v_{\mathrm{opt}} \neq v_{\text {diff }}$ effectively mixes dephasing and VCs, despite formally separating these effects into different terms. The commonly used phenomenological profiles are obtained by simplifying the collision operator. The speed-dependent hard-collision profile (SDHCP) [44] is obtained by replacing the billiard ball operator with the normalized hard-collision collision operator, $\hat{M}_{\mathrm{BB}} \equiv \hat{M}_{\mathrm{HC}}[19]$. The speed-dependent Voigt profile (SDVP) ignores VCs, $\hat{S}_{b a}=-\Gamma\left(v_{1}\right)-i \Delta\left(v_{1}\right)$, and the Voigt profile is obtained by replacing the speed-dependent broadening and shift in the SDVP with their thermally averaged values, $\Gamma\left(v_{1}\right) \equiv \Gamma_{0}$ and $\Delta\left(v_{1}\right) \equiv \Delta_{0}$.

The $\hat{M}_{\mathrm{HC}}$ operator does not depend on the interaction potential between the absorber and the perturber. Instead, it randomly selects the postcollision velocity from the MaxwellBoltzmann distribution, $f_{\mathrm{MB}}\left(\vec{v}_{1}\right)$, regardless of the initial velocity. This simple model enables a nearly analytical solution of Eq. (4) but fails to reproduce line shapes exhibiting a strong influence of VCs, such as molecular hydrogen transitions [45] - especially when hydrogen is perturbed by much heavier atoms or molecules. Moreover, using the hard-collision model to fit experimental spectra results in $v_{\text {opt }}$ values that scale nonlinearly with the pressure, which is unphysical. The billiard ball (hard-sphere) operator, $\hat{M}_{\mathrm{BB}}$, approximates the actual interaction potential by a hard-sphere potential. The diameter of the hard-sphere potential is set by the distance, $R_{\sigma}$, between the colliding species corresponding to $V_{0}\left(R_{\sigma}\right)=\langle E\rangle$, where $V_{0}$ is the isotropic part of the potential and $\langle E\rangle$ is the mean collision energy. The $\hat{M}_{\mathrm{BB}}$ operator takes into account the mass ratio of the colliding pair, the initial velocity, and 
the scattering angle. Most importantly, the SDBBP has been shown to reproduce experimental spectra in which VCs play a dominant role and to return physically meaningful line-shape parameters [18]. Recently, it was shown that the SDHCP can be used to approximate the SDBBP by replacing $v_{\mathrm{opt}}$ with $\beta\left(\chi=v_{\mathrm{opt}} / \Gamma_{D}, \alpha\right) v_{\mathrm{opt}}$ [36], where $\Gamma_{D}$ is the Doppler halfwidth and $\alpha=m_{2} / m_{1}$ is the perturber-to-absorber mass ratio. This $\beta$-corrected profile is denoted $\beta$ SDHCP in the following.

The prescription for calculating $\Gamma_{0}, \Delta_{0}$, and $v_{\mathrm{opt}}$ is given by the generalized Hess method (GHM) in terms of two collision integrals [37],

$$
\begin{aligned}
\omega_{A} & =\operatorname{Tr}\left\langle T_{b a}^{(q) n \dagger} \hat{S}\left(\rho^{(0)} T_{b a}^{(q) n}\right)\right\rangle_{1}=\Gamma_{0}+i \Delta_{0}, \\
\omega_{R} & =\frac{2}{3} \operatorname{Tr}\left\langle\vec{W}_{1} T_{b a}^{(q) n^{\dagger}} \hat{S}\left(\rho^{(0)} \vec{W}_{1} T_{b a}^{(q) n}\right)\right\rangle_{1} \\
& =\frac{2}{3} M_{2} \operatorname{Tr}\left\langle\vec{W}_{r} T_{b a}^{(q) n \dagger} \hat{S}\left(\rho^{(0)} \vec{W}_{r} T_{b a}^{(q) n}\right)\right\rangle_{r}+M_{1} \omega_{A} \\
& =v_{\text {diff }}^{\mathrm{rad}}+M_{1}\left(\Gamma_{0}+i \Delta_{0}\right),
\end{aligned}
$$

where Eq. (7) defines $v_{\text {diff }}^{\text {rad }}$. In Eqs. (6) and (7), $M_{i}=m_{i} /\left(m_{1}+\right.$ $\left.m_{2}\right),\langle\ldots\rangle_{1}$ denotes averaging over the Maxwell-Boltzmann distribution of the active molecule, $\langle\ldots\rangle_{r}$ denotes averaging over the relative motion, $\hat{S}$ is the collision operator for the full density matrix, and $\rho^{(0)}$ is the equilibrium density matrix for the absorber,

$$
\left[\rho^{(0)}\right]_{b a}=\delta_{a, b}\left(2 j_{a}+1\right) e^{-E_{j a} / k_{B} T} / Z_{\mathrm{rot}},
$$

where $k_{B}$ is the Boltzmann constant, $T$ is the temperature, and $Z_{\text {rot }}$ is the rotational partition function. The reduced velocities, $\vec{W}_{1}, \vec{W}_{r}$, are given by

$$
\begin{gathered}
\vec{W}_{1}=\left(\frac{m_{1}}{2 k_{B} T}\right)^{1 / 2} \vec{v}_{1}=\vec{W}_{r}+\vec{W}_{\mathrm{CM}}, \\
\vec{W}_{r}=\left(\frac{\mu_{12}}{2 k_{B} T}\right)^{1 / 2}\left(\vec{v}_{1}-\vec{v}_{2}\right), \\
\vec{W}_{\mathrm{CM}}=\left(\frac{1}{2\left(m_{1}+m_{2}\right) k_{B} T}\right)^{1 / 2}\left(m_{1} \vec{v}_{1}+m_{2} \vec{v}_{2}\right),
\end{gathered}
$$

where $\mu_{12}=m_{1} m_{2} /\left(m_{1}+m_{2}\right)$ and $\vec{v}_{2}$ is the velocity of the perturber. $\hat{S}$ depends only on the relative motion of the absorber-perturber pair, associated with $\vec{W}_{r}$ [38]. Hence, one can substitute Eq. (9) into Eq. (7) and integrate out the centerof-mass motion to split $\omega_{R}$ into the velocity-changing part, $v_{\text {diff }}^{\text {rad }}$, and the dephasing part, $M_{1}\left(\Gamma_{0}+i \Delta_{0}\right)$.

In order to calculate the HTP and SDBBP, we require the complex Dicke parameter, $v_{\text {opt }}$, which is related to the GHM quantities by

$$
v_{\mathrm{opt}}=\omega_{R}-\omega_{A}=v_{\mathrm{diff}}^{\mathrm{rad}}-M_{2}\left(\Gamma_{0}+i \Delta_{0}\right) .
$$

Equation (12) is formally similar to a commonly adopted formula for $v_{\mathrm{opt}}[17,44]$ :

$$
v_{\mathrm{opt}}=v_{\mathrm{diff}}-\eta\left(\Gamma_{0}+i \Delta_{0}\right) .
$$

Here, $\eta$ is the "correlation parameter" that has to be fitted to the experiment. Interpreting $\eta$ as the fractional contribution of dephasing to $v_{\mathrm{opt}}$, the GHM $[37,46]$ shows that it is fixed for a given pair of absorber-perturber species; see Eq. (12). Moreover, in the GHM the mass-diffusion contribution to $v_{\mathrm{opt}}$, $v_{\text {diff }}^{\text {rad }}$, is distinct from $v_{\text {diff }}$ and for CO-Ar it was found to be significantly larger than $v_{\text {diff }}[23]$.
The collision integrals in Eq. (12) are related to spectroscopic cross sections,

$$
\begin{gathered}
\omega_{A}=n_{2}\left\langle v_{r}\right\rangle\left\langle\sigma_{\lambda=0}^{q}\left(v_{a} j_{a} \rightarrow v_{b} j_{b} ; E\right)\right\rangle_{r}, \\
v_{\text {diff }}^{\mathrm{rad}}=\frac{2}{3} n_{2} M_{2}\left\langle v_{r}\right\rangle\left\langle x \sigma_{\lambda=1}^{q}\left(v_{a} j_{a} \rightarrow v_{b} j_{b} ; E\right)\right\rangle_{r},
\end{gathered}
$$

where $\left\langle v_{r}\right\rangle=\sqrt{8 k_{B} T / \pi \mu_{12}}$ is the mean relative speed, $E=$ $\mu_{12} v_{r}^{2} / 2$ is the initial kinetic energy, $x=E / k_{B} T$ is the reduced initial kinetic energy, $\lambda$ is the rank of the velocity tensor associated with the cross section, and $n_{2}$ is the concentration of the perturbers. The line shapes depend on the pressure through the ideal-gas law relation, $n_{2}=p / k_{B} T$. The rules for evaluating preceding thermal averages of cross sections and the expressions for these cross sections in terms of $S$-matrix elements in total angular momentum representation are given in Appendix A, Eqs. (A1)-(A3). It is worth pointing out that, in contrast to $v_{\text {diff }}, v_{\text {diff }}^{\text {rad }}$ can be a complex number. This is because $v_{\text {diff }}$ is calculated for collisions on a single interaction potential but $v_{\text {diff }}^{\text {rad }}$ involves collisions on the potential in the $(v, j)=\left(v_{a}, j_{a}\right)$ state and in the $\left(v_{b}, j_{b}\right)$ state; see Eq. (A1). Therefore, $v_{\text {opt }}$ is a complex quantity both due to this factor and due to the contribution from $\Delta_{0}$. The imaginary part of $v_{\text {opt }}$ induces asymmetry in the profile.

The speed-dependent pressure broadening and shift, $\Gamma\left(v_{1}\right)$ and $\Delta\left(v_{1}\right)$, are obtained by averaging the $\sigma_{\lambda=0}^{q}\left(v_{a} j_{a} \rightarrow\right.$ $\left.v_{b} j_{b} ; E\right)$ cross section over the conditional distribution $f_{\mathrm{MB}}\left(v_{r} \mid v_{1}\right)$ of the relative velocity with respect to the absorber velocity $[47,48]$ :

$$
\begin{aligned}
\Gamma\left(v_{1}\right)+i \Delta\left(v_{1}\right)= & n_{2} \int_{0}^{\infty} \mathrm{d} v_{r} v_{r} f_{\mathrm{MB}}\left(v_{r} \mid v_{1}\right) \\
& \times \sigma_{\lambda=0}^{q}\left(v_{a} j_{a} \rightarrow v_{b} j_{b} ; E=\mu_{12} v_{r}^{2} / 2\right) .
\end{aligned}
$$

In order to fit the experimental line shapes, $\Gamma\left(v_{1}\right)$ is commonly approximated with the quadratic speed dependence [49],

$$
\Gamma\left(v_{1}\right)=\Gamma_{0}+\Gamma_{2}\left(\frac{v_{1}^{2}}{v_{p}^{2}}-\frac{3}{2}\right),
$$

where $v_{p}=\sqrt{2 k_{B} T / m_{1}}$ is the most probable speed of the absorber. In particular, the SDHCP with quadratic speed dependence (qSDHCP) is the HTP $[17,50]$. We determine $\Gamma_{2}$ from the $a b$ initio dependence by requiring the derivatives of both the $a b$ initio and the quadratic speed dependence to be equal at $v_{1}=v_{p}[23]$ :

$$
\Gamma_{2}=\left.\frac{v_{p}}{2} \frac{\mathrm{d}}{\mathrm{d} v_{1}} \Gamma\left(v_{1}\right)\right|_{v_{1}=v_{p}} .
$$

The same formulas, Eqs. (17) and (18), mutatis mutandis, apply for $\Delta\left(v_{1}\right)$.

\section{RESULTS}

\section{A. Computational details}

In this work we use two fully $a b$ initio Ar-CO potentials: the Sumiyoshi and Endo [51] potential (henceforth Sumiyoshi potential) and a new potential calculated by us. The former was previously used by $\mathrm{Ngo}$ and Hartmann [7] for classical molecular dynamics simulations of $\mathrm{CO}-\mathrm{Ar}$ 0-3 line shapes 
[7] and by us for calculations of CO-Ar 0-1 line shapes [23]. The potentials are described and compared in more detail in Appendix B. Both potentials were expanded in the basis of Legendre polynomials up to 10th order and averaged over the $\mathrm{CO}$ wave function in $(v, j)=(0,0)$ and $(3,0)$ states. The difference at the minimum of the well between the Sumiyoshi potential averaged over the $(0,0)$ state and the $(0,14)$ state is $0.05 \mathrm{~cm}^{-1}$. Given the well depth of $107.1 \mathrm{~cm}^{-1}$, this indicates negligible centrifugal distortion. In order to assess the accuracy of potentials, we have performed full calculations of the line-shape parameters and profiles, as described in the following paragraphs, for the $\mathrm{P}(9)$ line for both potentials. Comparison with the experimental line shapes indicated that the Sumiyoshi potential provides better agreement, therefore it was used for all subsequent calculations and to obtain the results presented in Sec. III.

The $S$ matrices were obtained by numerically solving the close-coupled equations [52,53] with the log-derivative propagator [54,55] as implemented in the MOLSCAT code $[56,57]$. The close-coupling equations were propagated for intermolecular distances from 2 to $26 \AA$ or to the farthest classical turning point, whichever distance was larger. The $S$ matrices were calculated at kinetic energies up to $1700 \mathrm{~cm}^{-1}$ at 278 points, of which 247 were below $110 \mathrm{~cm}^{-1}$. The denser grid at lower energies was adopted to accurately probe the narrow Ar-CO resonances contributing to pressure shift cross sections. The upper limit of integration of thermal averages, Eqs. (14) and (15), was set by the 0.9999 quantile of the $x^{2} e^{-x}$ distribution, Eq. (A3). The maximum $v_{1}$ value at which $\Gamma\left(v_{1}\right)$ and $\Delta\left(v_{1}\right)$ were calculated was set by the 0.999 quantile of the Maxwell-Boltzmann speed distribution, and the upper limit of the speed-dependent average, Eq. (16), was set by the 0.995 quantile of the conditional distribution $f_{\mathrm{MB}}\left(v_{r} \mid v_{1}\right)$. Cross sections for energies beyond $1700 \mathrm{~cm}^{-1}$ were obtained from simple extrapolations with the $f(E)=a / E+b$ function, where $a, b$ were fitted parameters. The extrapolated integrands of Eqs. (14) and (15) were interpolated with cubic B splines and integrated using analytical formulas.

\section{B. Comparison of $a b$ initio and experimental line profiles}

In order to directly verify our calculations, we present in Fig. 1 a comparison between line profiles of the $\mathrm{P}(9)$ line measured with a comb-assisted cavity ringdown spectrometer [32] (top row), simulated based on ab initio calculations [rows (a)-(g)] and fitted to the measurements [row (h)]. Most of the $a b$ initio calculations were done with the Sumiyoshi potential [Figs. 1(a)-1(e), 1(g)]. The presented measurement data and fitted line-shape parameters are the same as in Ref. [32] and the reader is referred there for a detailed description of the experimental setup and procedure. At each pressure the frequency axis was shifted to the position of the line peak determined from the qSDHCP fit, i.e., by the value $v_{0}+\Delta_{0}$, where $v_{0}$ is the zero-pressure transition frequency. The plotted frequency span was limited to \pm 4 full-widths at half-maximum (FWHM) at each pressure. We selected the $\mathrm{P}(9)$ line due to the high SNR of the measurements of this line, allowing us to distinguish the influence of different collisional effects on the line shape. Conversely, we do not show the lowest experimental pressure line profile, 10 Torr, because its low SNR would prevent any meaningful analysis of the line shape. Figures 1(a)-1(h) show the differences between experimental line profiles and different models, scaled to the peak of the experimental profile at each pressure. The level of agreement between the experimental and the simulated line profiles is quantified with the root-mean-square difference between them, divided by the peak value of the experimental profile at each pressure. The calculated values are denoted $\epsilon$ in Fig. 1. The experimental SNR is equivalent to the $1 / \epsilon$ values for the qSDHCP fit [Fig. 1(h)] since the fit reproduces the spectra within the experimental noise. From lowest to highest pressure the SNR values are 1081, 1196, 1497, 1584. For the fully $a b$ initio profiles [Figs. 1(a)-1(f)], line intensities, transition frequencies, and baseline functions (linear slope and two etalons) were set to the values obtained from the qSDHCP multispectrum fit of the experimental data [32]; the collisional line-shape parameters, Eq. (5), were set to $a b$ initio values; and the Doppler width was determined from the temperature of the sample. The $\Gamma_{0}$-corrected $\beta$ SDHCP [Fig. 1(g)] was calculated with the fitted instead of the $a b$ initio $\Gamma_{0}$, but it is otherwise the same as the $\beta$ SDHCP [Fig. 1(d)].

The pressures of $50.02,99.69,299.78$, and 702.46 Torr correspond to the $\Gamma_{0} / \Gamma_{D}$ ratios of $0.43,0.86,2.6$, and 6.0 , respectively, for the $\mathrm{P}(9)$ line in Fig. 1. Given that $\Gamma_{D}$ and $\Gamma_{0}$ approximately add in quadrature $[58,59]$ to produce the total width, this indicates that Dicke narrowing should be prominent at the two lowest pressures. At 702.46 Torr the width of the line is dominated by pressure broadening and the reduction of the Doppler width by VCs is expected to have a negligible influence on the overall line width. The large residuals for the Voigt profile [Fig. 1(a)] demonstrate the well-established limitations of the profile [60], showing wshaped residuals characteristic of Dicke narrowing or speeddependent narrowing. The addition of the speed dependence of pressure broadening and shift to the model reduces the residuals by more than a factor of 2 [Fig. 1(b); note the scale change]. The description of the line shape is further improved by including the effect of VCs in the model, either with the hard-collision model [Fig. 1(c)] or with the billiard ball model [Figs. 1(d) and 1(e)].

The SDBBP [Fig. 1(e)] offers a clear improvement over the SDHCP [Fig. 1(c)]. As expected, the differences between the profiles fall off with increasing pressure as the Dicke effect diminishes in importance. In fact, the differences between the SDBBP and all the other speed-dependent profiles using the Sumiyoshi potential [Figs. 1(b)-1(d)] are minor at 702.36 Torr and all these profiles are broader than the experimental line. Their line widths cannot be reduced by collisional narrowing because at this pressure and a $\Gamma_{0} / \Gamma_{D}$ ratio of 6.0 the influence of VCs on the line shape is minimal. This point is simply demonstrated by calculating, for example, the SDHCP with $v_{\mathrm{opt}}$ equal to 10 times the $a b$ initio $v_{\mathrm{opt}}$. In this case the profile at 702.36 Torr is as broad as it was before but at 50.02 Torr the line is too narrow with $\epsilon \approx 8 \%$. This is another way of saying that we have reached the pressure-broadening regime, in which any reasonable description of VCs in the collision operator would produce a similar width. The ab initio lines are too broad because the calculated $\Gamma_{0}$ is larger than the experimental one. This conclusion is confirmed by the 


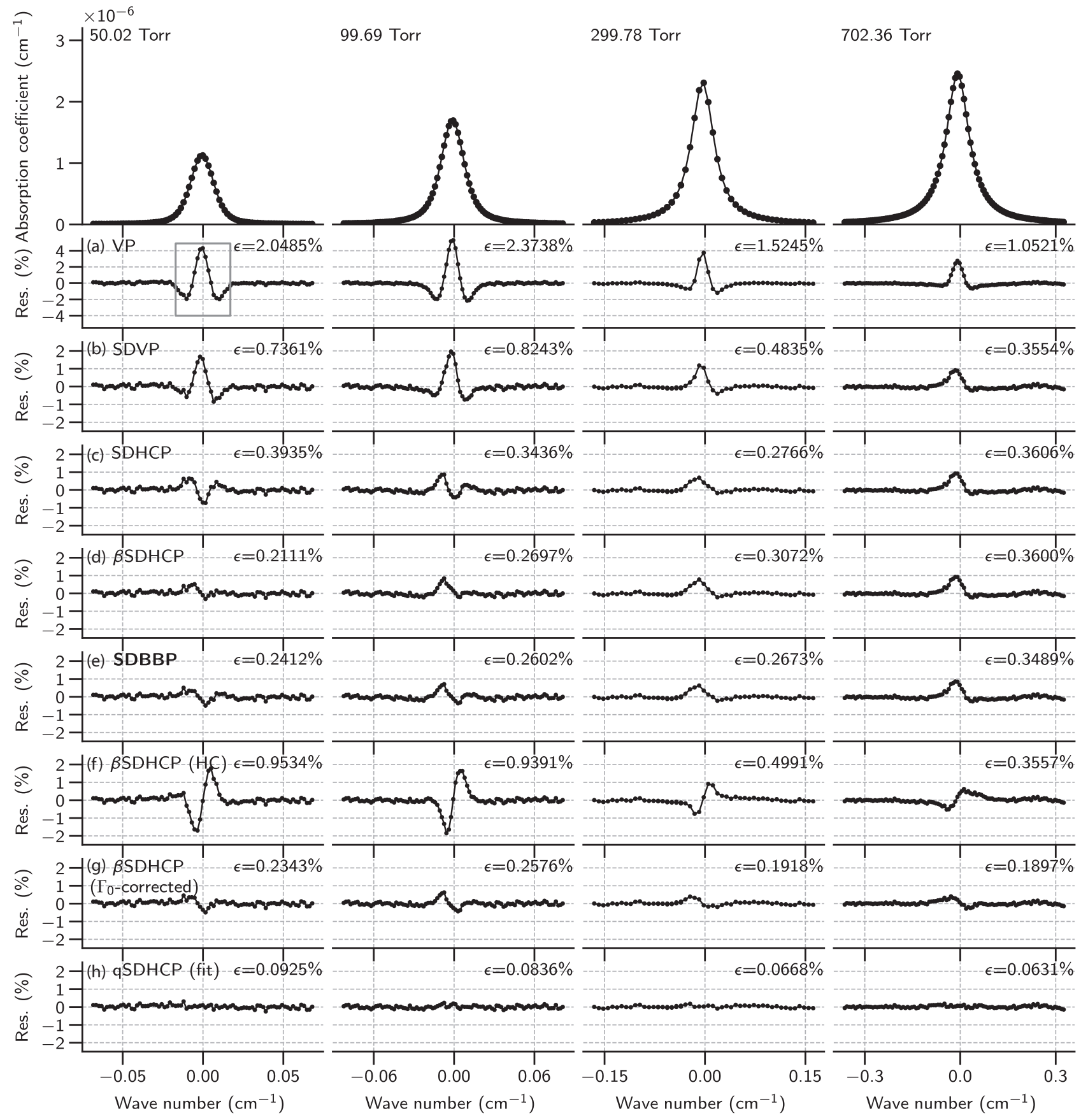

FIG. 1. Experimental line shapes of the $\mathrm{P}(9)$ line (top panel) and relative differences (residuals) between the measured and ab initio line-shape profiles (a)-(g) and the fitted profile (h). In each case the plotted range is the $\pm 4 \times$ FWHM region around the experimental line peak. Rows (a)-(e) show ab initio line-shape profiles of increasing sophistication; see Sec. II for an explanation of the acronyms. Row (e) is the most sophisticated and physically justified model evaluated for fully ab initio parameters. Row (f) is the $\beta$ SDHCP, the same as row (d), but with the line-shape parameters based on our potential. Row $(\mathrm{g})$ is the $\beta \mathrm{SDHCP}$, the same as row (d), but with the $\Gamma_{0}$ values from the qSDHCP fit [32]. Finally, row (h) shows the qSDHCP with all the parameters obtained from a multispectrum fit to the experiment. The $\epsilon$ values for each residual are the relative root-mean-square errors expressed as percentages, which were calculated in the \pm FWHM range marked in row (a), 50 Torr, by the gray rectangle.

significantly lower $\epsilon$ values at 299.78 and 702.36 Torr for $\beta$ SDHCP [Fig. $1(\mathrm{~g})$ ] evaluated with the fitted $\Gamma_{0}$.

The remaining residuals in Fig. 1(g) at all but the lowest pressure are strongly asymmetric. Within the binary collision impact approximation [61], asymmetric residuals can be caused by an error in the thermally averaged $\left(\Delta_{0}\right)$ or speed-dependent $\left(\Delta_{2}\right)$ shift or by mixing of dephasing and VCs $\left(\operatorname{Im} v_{\text {opt }}\right)$. As we show further in Sec. III C, the latter two factors are too weak to produce the asymmetry in Fig. 1(g), so we conclude that the asymmetry is caused by an error in the $a b$ initio $\Delta_{0}$. Interestingly, at 50.02 Torr the shape of the residuals [Fig. 1(e)] indicates that the $a b$ initio profile is too 
TABLE I. Ab initio and fitted line-shape parameters. Experimental parameters were determined from multispectrum qSDHCP fits to comb-assisted cavity ringdown spectroscopy measurements [32]. Ab initio parameters were calculated following the procedure described in Sec. II and Ref. [23] and were based on the Sumiyoshi potential [51] except the "(HC)" parameters for the P(9) line, which were based on our potential. Differences between experimental and theoretical values are given as percentages and the temperature is given in kelvins. All other values are given in $10^{-3} \mathrm{~cm}^{-1} / \mathrm{atm}$. The lowercase quantities $\left(\gamma_{0}, \delta_{0}, \gamma_{2}, \delta_{2}\right)$ are pressure-independent counterparts of uppercase line-shape parameters, i.e., $\gamma_{0} \equiv \Gamma_{0} / p$, etc., and $\tilde{v}_{\text {opt }} \equiv v_{\text {opt }} / p$.

\begin{tabular}{|c|c|c|c|c|c|c|c|c|}
\hline Line & $T(\mathrm{~K})$ & & $\gamma_{0}$ & $\delta_{0}$ & $\gamma_{2}$ & $\delta_{2}$ & $\operatorname{Re} \tilde{\nu}_{\text {opt }}$ & $\operatorname{Im} \tilde{v}_{\text {opt }}$ \\
\hline $\mathrm{P}(2)$ & 295.6 & $\begin{array}{l}\text { Experiment } \\
\text { Theory } \\
\text { Difference }\end{array}$ & $\begin{array}{l}65.76(29) \\
66.00 \\
-0.4\end{array}$ & $\begin{array}{l}-4.976(26) \\
-4.85 \\
2.6\end{array}$ & $\begin{array}{c}7.07(15) \\
8.51 \\
-20.4\end{array}$ & $\begin{array}{c}- \\
-0.67 \\
-\end{array}$ & $\begin{array}{c}4.7(4) \\
8.63 \\
-85.1\end{array}$ & $\begin{array}{r}- \\
-0.28 \\
-\end{array}$ \\
\hline $\mathrm{P}(4)$ & 295.7 & $\begin{array}{l}\text { Experiment } \\
\text { Theory } \\
\text { Difference }\end{array}$ & $\begin{array}{l}58.07(25) \\
57.95 \\
0.2\end{array}$ & $\begin{array}{l}-7.302(33) \\
-7.10 \\
2.8\end{array}$ & $\begin{aligned} & 7.95(9) \\
& 8.41 \\
- & 5.8\end{aligned}$ & $\begin{array}{c}- \\
-0.07 \\
-\end{array}$ & $\begin{array}{c}4.39(25) \\
10.15 \\
-131.3\end{array}$ & $\begin{array}{l}1.81(12) \\
0.80 \\
55.9\end{array}$ \\
\hline $\mathrm{P}(6)$ & 296.1 & $\begin{array}{l}\text { Experiment } \\
\text { Theory } \\
\text { Difference }\end{array}$ & $\begin{array}{l}51.95(21) \\
52.06 \\
-0.2\end{array}$ & $\begin{array}{l}-8.406(35) \\
-8.35 \\
0.7\end{array}$ & $\begin{array}{l}7.13(6) \\
7.00 \\
1.8\end{array}$ & $\begin{array}{c}- \\
-0.37 \\
-\end{array}$ & $\begin{array}{c}3.80(17) \\
10.32 \\
-171.4\end{array}$ & $\begin{array}{l}1.32(8) \\
1.09 \\
17.6\end{array}$ \\
\hline $\mathrm{P}(9)$ & 295.7 & $\begin{array}{c}\text { Experiment } \\
\text { Theory } \\
\text { Difference } \\
\text { Theory (HC) } \\
\text { Difference (HC) }\end{array}$ & $\begin{array}{l}47.52(21) \\
47.87 \\
-0.7 \\
47.47 \\
0.1\end{array}$ & $\begin{array}{l}-8.81(4) \\
-8.71 \\
1.1 \\
-9.27 \\
-5.3\end{array}$ & $\begin{aligned} & 5.46(10) \\
& 5.60 \\
- & 2.4 \\
& 5.71 \\
- & 4.4\end{aligned}$ & $\begin{array}{l}- \\
-0.55 \\
- \\
-0.65 \\
-\end{array}$ & $\begin{array}{c}5.30(20) \\
9.73 \\
-83.7 \\
9.65 \\
-82.2\end{array}$ & $\begin{array}{l}0.95(7) \\
0.90 \\
5.2 \\
0.90 \\
6.0\end{array}$ \\
\hline $\mathrm{P}(14)$ & 295.7 & $\begin{array}{c}\text { Experiment } \\
\text { Theory } \\
\text { Difference }\end{array}$ & $\begin{array}{l}45.66(21) \\
45.04 \\
1.3\end{array}$ & $\begin{array}{l}-8.97(4) \\
-8.93 \\
0.4\end{array}$ & $\begin{array}{l}6.80(9) \\
4.89 \\
28.1\end{array}$ & $\begin{array}{c}- \\
-0.45 \\
-\end{array}$ & $\begin{array}{c}2.97(22) \\
9.82 \\
-230.4\end{array}$ & $\begin{array}{r}- \\
0.74 \\
-\end{array}$ \\
\hline
\end{tabular}

narrow, in addition to being shifted by the $\Delta_{0}$ error, similarly to the case of the uncorrected SDHCP [Fig. 1(c)]. We saw the same effect in the fundamental band of CO-Ar, where the $v_{\text {opt }}$ retrieved from an SDBBP fit to the $\mathrm{P}(2)$ line was smaller [20] than the value predicted by the GHM [23]. There are several viable explanations for this effect. It is possible that the SDBBP approximation of the actual interaction potential with a hard-sphere potential overestimates the narrowing effect of VCs. Wcisło and Ciuryło [62] investigated this problem by comparing line shapes produced by hard-sphere scattering and by scattering on $r^{-q}$-type potentials. They showed that the latter actually produces narrower instead of broader line shapes, but it is not clear whether an $r^{-q}$-type potential in fact describes the effect of VCs more realistically. One could also attempt to improve the line-shape model by including the speed dependence of $v_{\text {opt }}$, but in our previous work we have found that doing so also produces narrower line shapes [23]. As another option, the value of $v_{\mathrm{opt}}$ obtained from the GHM could be too large. In fact, in his original paper [46] Hess considered a higher-order correction to $v_{\text {opt }}$ related to the viscosity, which, at least for $\mathrm{H}_{2}-\mathrm{H}_{2}$ interaction, was estimated to reduce the value of $v_{\text {opt }}$. We return to this problem in Sec. IIIC where we compare fitted and $a b$ initio $v_{\mathrm{opt}}$ values.

In Fig. 1(f), we show the residuals of the $\beta$ SDHCP evaluated with line-shape parameters based on our potential. The strongly asymmetric residuals over the whole pressure range are caused by a significant error in pressure shift, which also occurs for the rest of the lines considered in this article (see Appendix B). Interestingly, the $\epsilon$ value for this potential at 702.36 Torr is lower than for the Sumiyoshi one. This is because our $\Gamma_{0}$ is more accurate than that of Sumiyoshi, which is not apparent under other conditions where the $\Delta_{0}$ error dominates. The foregoing analysis underlines the importance of employing an accurate interaction potential in $a b$ initio line-shape modeling.

The $\beta$ SDHCP residuals [Fig. 1(d)] show the first experimental verification of the $\beta$ correction generalized to molecules other than $\mathrm{H}_{2}$, confirming its utility on actual data. These results confirm the viability of generating the full set of HTP parameters in a fully ab initio manner [23] and show the $\beta$ correction to be a crucial component of the proposed procedure.

\section{Comparison of $a b$ initio and fitted line-shape parameters}

Table I reports the calculated line-shape parameters and compares them with the experimentally fitted ones. The theoretical pressure-independent parameters $-\gamma_{0}, \delta_{0}, \gamma_{2}, \delta_{2}$, and $\tilde{v}_{\text {opt }}$, in units of $\mathrm{cm}^{-1} / \mathrm{atm}$-were obtained by multiplying Eqs. (14), (15), and (16) by the conversion factor, $\left(n_{0} / n_{2}\right)\left(T_{0} / T\right)(2 \pi c)^{-1}$, where $n_{0}$ is the Loschmidt constant, $T_{0}=273.15 \mathrm{~K}$, and $c$ is the speed of light in vacuum. The uncertainties of the experimental values are the total combined uncertainties from [32]. As the error of the ab initio $\gamma_{0}$ and $\delta_{0}$, we simply take their difference from experimental values. In principle, disagreement between the $a b$ initio and the fitted $\gamma_{0}$ and $\delta_{0}$ does not imply that the $a b$ initio values are inaccurate [18], but we have already established in Sec. III B that no other factor can account for the residuals we observe at high pressure. Overall, the $a b$ initio $\gamma_{0}$ values have subpercent errors, with the exception of the $\mathrm{P}(14)$ line, and $\delta_{0}$ values are accurate up to a few percent. This level of accuracy compares favorably with requantized CMDS calculations of $\gamma_{0}$ in the same band and based on the same potential. In that case, $\gamma_{0}$ values for $\mathrm{P}(1)$ and $\mathrm{P}(17)$ lines were found to differ from 
experimental values by $13.8 \%$ and $4.6 \%$, respectively [7]. We estimate the absolute errors of our calculated $\gamma_{2}$ and $\delta_{2}$ to be of the same order as the errors of $\gamma_{0}$ and $\delta_{0}$, respectively, namely, at the level of a few $10^{-4} \mathrm{~cm}^{-1} / \mathrm{atm}$.

Table I shows a large difference between ab initio-based and fitted values of $\gamma_{2}$ and $\operatorname{Re} \tilde{v}_{\text {opt }}$. Considering the excellent agreement between $a b$ initio and experimental line shapes in Fig. 1, these differences cannot be wholly attributed to an error in our calculations or in our procedure. There are several additional factors to consider: first, both speed-dependent broadening and VCs narrow the line shapes, which makes the parameters associated with these effects strongly negatively correlated in the fit; second, the quadratic approximation, Eqs. (17) and (18), models the actual speed dependence of $\gamma\left(v_{1}\right)$ with limited accuracy; third, the $\beta$ correction decreases the value of $\operatorname{Re} \tilde{v}_{\text {opt }}$ and causes it to scale nonlinearly with pressure, but linear scaling was assumed in [32]. Considering these model errors, it is expected for the differences between $a b$ initio-based and fit parameters to surpass the nominal fit uncertainties. Nevertheless, it is desirable to identify the reason for these discrepancies, to quantify the different contributions, and to determine whether retroactively applying the $\beta$ correction will allow us to perform a meaningful comparison.

We estimate the uncertainty of ab initio $\tilde{v}_{\text {opt }}$ by assuming the error of $\nu_{\mathrm{diff}}^{\mathrm{rad}} / p$ to be the same as that of $\gamma_{0}+i \delta_{0}$ and equal to the difference between the experimental and the $a b$ initio $\gamma_{0}+i \delta_{0}$. From the standard rule of uncertainty propagation for uncorrelated variables, this results in

$$
u\left(\operatorname{Re} \tilde{v}_{\text {opt }}\right)=u\left(\gamma_{0}\right) \sqrt{\left(1+M_{2}^{2}\right)},
$$

and analogously for Im $\tilde{v}_{\text {opt }}$. Figure 2 shows the comparison between $a b$ initio and fitted values of the complex Dicke parameter $\tilde{v}_{\text {opt }}$, Eq. (12). For comparison with the experiment, the $a b$ initio values and the SDBBP fit value were scaled by the $\beta$ correction. This phenomenological correction factor was determined only for real $\tilde{v}_{\text {opt }}$ and we do not apply it to the imaginary part of our $\tilde{v}_{\text {opt }}$. The thick vertical blue lines capped with error bars in Fig. 2(a) represent the range of values adopted by $\beta(\chi, \alpha) \operatorname{Re} \tilde{v}_{\text {opt }}$ in the range of $10-$ 700 Torr, and analogously for the open red rectangle, which was obtained from an SDBBP fit [20]. This pressure range corresponds to $\beta(\chi, \alpha)$ values between 0.73 and 0.89 . The fitted qSDHCP values (green crosses) are the same as in [32] and were obtained from multispectrum fits with the uncorrected qSDHCP. The comparison can still be affected by the errors of quadratic approximation, and to refine it we estimate the deviation of the actual speed dependence from the quadratic speed dependence. We do so by integrating the relative absolute-value difference between the $a b$ initio and the quadratic $\gamma\left(v_{1}\right)$ weighted by $f_{\mathrm{MB}}\left(v_{1}\right)$,

$$
\epsilon_{\gamma_{2}}=\frac{1}{\gamma_{0}} \int_{0}^{\infty} \mathrm{d} v_{1} f_{\mathrm{MB}}\left(v_{1}\right)\left|\gamma\left(v_{1}\right)-\gamma_{0}-\gamma_{2}\left(\frac{v_{1}^{2}}{v_{p}^{2}}-\frac{3}{2}\right)\right|,
$$

where $\gamma_{2}$ was determined from the $a b$ initio cross sections according to Eq. (18). The $\epsilon_{\gamma_{2}}$ values for different lines are listed in Table II. The clearest picture emerges for the $\mathrm{P}(9)$ line, for which $\gamma\left(v_{1}\right)$ is well approximated by the quadratic speed dependence and the value retrieved from the qSDHCP

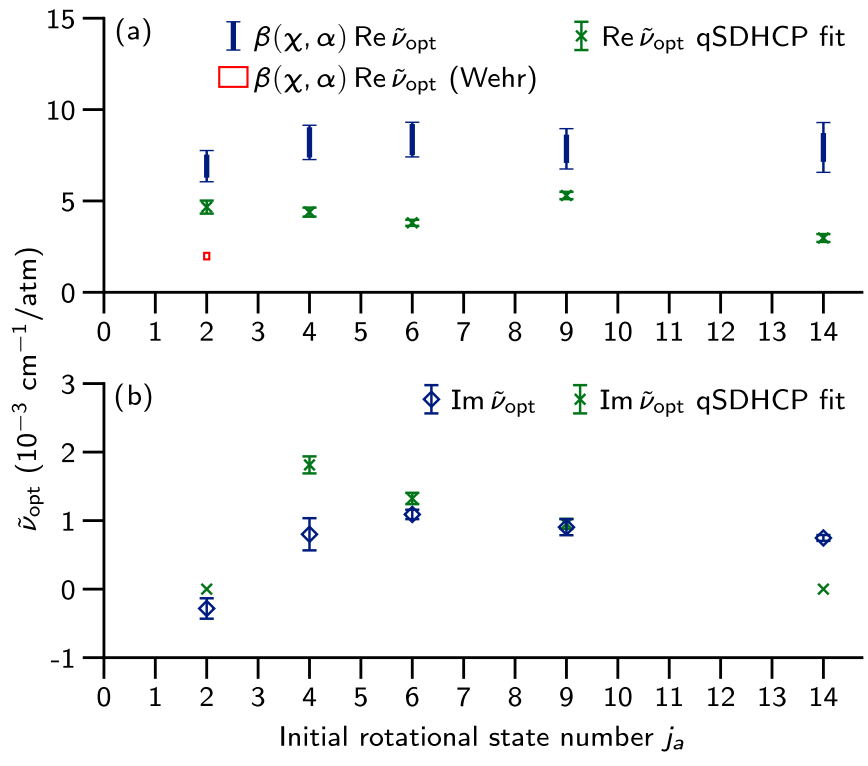

FIG. 2. Real part (a) and imaginary part (b) of the complex Dicke parameter $\tilde{v}_{\text {opt }}$, Eq. (12). Values are plotted as a function of the initial rotational state of the transition $\mathrm{P}\left(j_{a}\right)$. Thick blue lines capped with error bars (a) mark the range of values adopted by the $\beta$-corrected [36] ab initio $\operatorname{Re} \tilde{v}_{\mathrm{opt}}$ in the experimental pressure range, $10-700$ Torr. Analogously, the open red rectangle represents the $\beta$-corrected $\operatorname{Re} \tilde{v}_{\text {opt }}$ from [20]. Blue diamonds (b) show the ab initio $\operatorname{Im} \tilde{v}_{\text {opt }}$ values, and green crosses with error bars (a), (b) represent the values retrieved from fitting the qSDHCP to the experimental data.

fit closely matches the $a b$ initio-derived one (see Table I). Therefore, the difference between ( $\beta$-corrected) $\tilde{v}_{\text {opt }}$ 's for $\mathrm{P}(9)$ in Fig. 2 confirms the excessive narrowing shown in Fig. 1(d) and quantifies it in terms of the $\tilde{\nu}_{\text {opt }}$ parameter.

Continuing with the remaining lines, we clearly see the influence of the negative correlation between $\gamma_{2}$ and $\operatorname{Re} \tilde{v}_{\mathrm{opt}}$ for $\mathrm{P}(2)$ and $\mathrm{P}(14)$. For the $\mathrm{P}(2)$ line, $\gamma_{2}$ was underestimated and the fitted $\operatorname{Re} \tilde{v}_{\text {opt }}$ agrees better with the ab initio $\operatorname{Re} \beta(\chi, \alpha) \tilde{v}_{\text {opt }}$. The accuracy of the $\beta$ correction was established in Sec. III B, therefore we can also conclude that the difference between $\operatorname{Re} \beta(\chi, \alpha) \tilde{v}_{\text {opt }}$ from the SDBBP fit (red rectangle) and Re $\tilde{v}_{\text {opt }}$ from the qSDHCP fit is mostly caused by the incorrect speed dependence of broadening. For the $\mathrm{P}(14)$ line, the fit overestimated $\gamma_{2}$ (see Table I), which agrees with the correspondingly lower value of Re $\tilde{v}_{\text {opt }}$. The $\gamma_{2}$ values for $\mathrm{P}(4)$ and $\mathrm{P}(6)$ are encumbered by similar approximation errors as the $\mathrm{P}(2)$ line but the fitted values match the predicted ones closely. If the parameter correlation and the error of quadratic approximation were sufficient to explain the observed differences between the ab initio $\operatorname{Re} \beta(\chi, \alpha) \tilde{v}_{\text {opt }}$ and the fitted $\operatorname{Re} \tilde{v}_{\text {opt }}$, we would expect the fitted Re $\tilde{v}_{\text {opt }}$ for $\mathrm{P}(2)$ to be larger than the $a b$ initio one, the value for $\mathrm{P}(14)$ to be

TABLE II. Estimated errors of the quadratic approximation, Eq. (20), for CO-Ar second-overtone $\mathrm{P}\left(j_{a}\right)$ lines.

\begin{tabular}{lccccc}
\hline \hline & \multicolumn{5}{c}{ Line } \\
\cline { 2 - 6 } & $\mathrm{P}(2)$ & $\mathrm{P}(4)$ & $\mathrm{P}(6)$ & $\mathrm{P}(9)$ & $\mathrm{P}(14)$ \\
$\epsilon_{\gamma_{2}}$ & 0.027 & 0.025 & 0.013 & 0.006 & 0.004 \\
\hline \hline
\end{tabular}


lower, and the values for the remaining lines to be scattered around the predicted ones. Clearly, this is not the case and this analysis shows that excessive narrowing occurs for all the considered lines. Moreover, it demonstrates that the $\beta$ correction augmented with ab initio line-shape parameters can be used for retroactive data correction.

It is important to realize that the large difference between $a b$ initio and experimental $\operatorname{Re} \tilde{v}_{\text {opt }}$ values is not caused by an error in the interaction potential or in scattering calculations. The primary difficulty in obtaining $a b$ initio $\operatorname{Re} \tilde{v}_{\text {opt }}$ values, which allow us to accurately model experimental lines with the SDHCP or the SDBBP, lies in the strong model dependence of this parameter. This is illustrated by the $\beta$ correction, which for CO-Ar approximates the SDBPP with the SDHCP by scaling down $\operatorname{Re} \tilde{v}_{\text {opt }}$ to as little as $73 \%$ of its original value. This problem can be bypassed by numerically solving Eq. (4) with an exact collision operator [63], but this way is computationally expensive and would provide little physical insight.

The differences between fitted and $a b$ initio $\operatorname{Im} \tilde{v}_{\mathrm{opt}}$ values [Fig. 2(b)] reach at most $10^{-3} \mathrm{~cm}^{-1} / \mathrm{atm}$ and can be attributed to several factors. Foremost, the asymmetry of the peak in the considered lines is weak. For all the lines $\left|\delta_{2}\right|<10^{-3} \mathrm{~cm}^{-1} /$ atm, $\left|\operatorname{Im} \tilde{v}_{\text {opt }}\right|<2 \times 10^{-3} \mathrm{~cm}^{-1} / \mathrm{atm}$, and the estimated calculation uncertainty of a few $10^{-4} \mathrm{~cm}^{-1} / \mathrm{atm}$ represents a large relative uncertainty. Experimentally, for the $\mathrm{P}(2)$ and $\mathrm{P}(14)$ lines the asymmetry was below the noise level and for the remaining lines ignoring the asymmetry changed the $\epsilon$ values at the $10^{-5}$ level. Moreover, $a b$ initio calculations predict nonzero $\delta_{2}$ values for all the lines, yet the qSDCHP fit was incapable of retrieving both $\delta_{2}$ and $\operatorname{Im} \tilde{v}_{\text {opt }}$ at our noise level due to parameter correlation and likely due to imperfect modeling of $\Delta\left(v_{1}\right)$ with the quadratic speed dependence, Eq. (18). Additionally, unwanted etaloning in the setup could have spuriously affected the asymmetry of the peak and shifted the value of $\operatorname{Im} \tilde{v}_{\text {opt }}$. For these reasons, we do not consider the apparent disagreement between the $a b$ initio and the experimental Im $\tilde{v}_{\text {opt }}$ to be physically relevant. Some of these issues could be mitigated by fixing $\delta_{2}$ or Im $\tilde{v}_{\text {opt }}$ to the $a b$ initio value and repeating the multispectrum fit to retrieve the value of the other parameter. However, this does not address the weakness of the asymmetry relative to the noise level and to residual etalons, which would make any conclusions based on such a fit uncertain. It would be desirable to confront experimental speed-dependent and correlation asymmetry with $a b$ initio predictions, but preferably based on less noisy spectra and in a system in which these effects are more prominent.

The results of the calculations presented in the article are available in the Supplemental Material [64], including the spectroscopic cross sections $\sigma_{\lambda=0}^{1}, \sigma_{\lambda=1}^{1}$, the line-shape parameters $\gamma_{0}, \delta_{0}, \gamma_{2}, \delta_{2}, \tilde{v}_{\text {opt }}$ in the $10-800 \mathrm{~K}$ temperature range, and our Ar-CO potential energy surface.

\section{CONCLUSIONS}

We achieved a subpercent agreement in a direct comparison between $a b$ initio and experimental line shapes of $\mathrm{CO}$-Ar, without adjusting any of the line-shape parameters to the experiment (see Sec. III B). These results are the most accurate $a b$ initio calculations of collisional line shapes for an atmospherically relevant species. They set down a new benchmark for comparisons between theoretical and experimental molecular line shapes. The remaining discrepancies were predominantly caused by the limited accuracy of the calculated $\gamma_{0}$ and $\delta_{0}$, which was attributed to the interaction potential. Nevertheless, four of the five reported $\gamma_{0}$ values are accurate to better than a percent and the $\delta_{0}$ values are accurate within a few percent (see Sec. III C). The $\beta$ correction to the hard-collision profiles allowed us to faithfully reproduce the SDBBP in a fraction of the time required to calculate the latter and validate the procedure for generating HTP line-shape parameters proposed in [23].

The analysis of the line-shape residuals in Sec. III B has shown that our procedure overestimates the Dicke narrowing for the $\mathrm{P}(9)$ line. This observation was extended to all the other considered lines by comparing the fitted and calculated lineshape parameters in Sec. III C. We have considered several possible explanations for this effect, but providing a definite answer will require further investigations. The presented analysis also demonstrates that the $\beta$ correction facilitates a physically meaningful interpretation of fitted qSDHCP parameters and their comparison with SDBBP and $a b$ initio line-shape parameters.

A new potential energy surface for Ar-CO interaction was calculated and compared to the Sumiyoshi surface [51] (see Appendix B). The differences in pressure-broadening parameters based on the two potentials were found to be caused by different total inelastic cross sections. For the shifts, the differences in vibrational dependence of the potentials were identified as the reason for the discrepancies.

\section{ACKNOWLEDGMENTS}

This research was supported by National Science Centre, Poland, Projects No. 2016/21/N/ST2/00334 and No. 2016/23/B/ST2/00730. G.K. was supported by National Science Centre, Poland, scholarship 2017/24/T/ST2/00242. H.C.'s contribution was financed by the National Science Centre, Poland, within OPUS 8 Project No. 2014/15/B/ST4/04551. P.W.'s contribution was supported by the National Science Centre, Poland, through Project No. 2018/31/B/ST2/00720. M.S.'s contribution was supported by the National Science Centre, Poland, through Project No. 2017/26/D/ST2/00371 and by the A Next-Generation Worldwide Quantum Sensor Network with Optical Atomic Clocks project carried out within the TEAM IV Programme of the Foundation for Polish Science cofinanced by the European Union under the European Regional Development Fund. The project is supported by the French-Polish PHC Polonium program (Project No. 42769ZK for the French part). The project is cofinanced by the Polish National Agency for Academic Exchange under the PHC Polonium program (dec. PPN/X/PS/318/2018). The research is part of the program of the National Laboratory FAMO (KL FAMO) in Torun, Poland, and is supported by a subsidy from the Polish Ministry of Science and Higher Education. Some of the calculations were carried out using resources provided by Wroclaw Centre for Networking and Supercomputing [65] Grant No. 294. 


\section{APPENDIX A: CROSS SECTIONS AND THERMAL AVERAGING}

The cross section for velocity change of an optical coherence for an absorption line $v_{a} j_{a} \rightarrow v_{b} j_{b}$ is given by $[25,37,66,67]$

$$
\begin{aligned}
& \sigma_{\lambda=1}^{q}\left(v_{a} j_{a} \rightarrow v_{b} j_{b} ; E\right) \\
& =\frac{\pi}{k^{2}} \sum_{\substack{J_{a}, J_{b} \\
l_{a}, l_{a}^{\prime}, l_{b}, l_{b}^{\prime}}}\left[J_{a}\right]\left[J_{b}\right] \sqrt{\left[l_{a}\right]\left[l_{a}^{\prime}\right]\left[l_{b}\right]\left[l_{b}^{\prime}\right]} \\
& \quad \times\left(\begin{array}{ccc}
l_{a} & l_{b} & \lambda \\
0 & 0 & 0
\end{array}\right)\left(\begin{array}{ccc}
l_{a}^{\prime} & l_{b}^{\prime} & \lambda \\
0 & 0 & 0
\end{array}\right)\left[\begin{array}{cccc}
j_{b} & j_{b} & l_{a} & l_{a}^{\prime} \\
j_{a} & l_{b} & j_{a} & l_{b}^{\prime} \\
q & J_{a} & J_{b} & \lambda
\end{array}\right] \\
& \quad \times i^{l_{a}^{\prime}-l_{a}-l_{b}^{\prime}+l_{b}}\left(\delta_{l_{a} l_{a}^{\prime}} \delta_{l_{b} l_{b}^{\prime}}-S_{v_{b} j_{b} l_{b}^{\prime}, v_{b} j_{b} l_{b}}^{J_{b}} S_{v_{a} j_{a} l_{a}, v_{a} j_{a} l_{a}}\right),
\end{aligned}
$$

where $k^{2}=2 \mu_{12} E / \hbar^{2},[x]=(2 x+1),\{: \because:\}$ is the 6-j symbol,

$[\cdots \cdots$.$] is the 12-j symbol of the second kind [68], \lambda$ is the rank of the velocity tensor associated with the cross section, $J_{a}, J_{b}$ are the total angular momenta, and $l_{a}, l_{a}^{\prime}, l_{b}, l_{b}^{\prime}$ are the orbital angular momenta. The primed indices correspond to postcollision quantities, and unprimed to precollision quantities.

The pressure-broadening and shift cross section for an absorption line $v_{a} j_{a} \rightarrow v_{b} j_{b}$ is given by

$$
\begin{aligned}
\sigma_{\lambda=0}^{q}( & \left.v_{a} j_{a} \rightarrow v_{b} j_{b} ; E\right) \\
= & \frac{\pi}{k^{2}} \sum_{J_{a}, J_{b}, l, l^{\prime}}\left[J_{a}\right]\left[J_{b}\right](-1)^{l-l^{\prime}} \\
& \times\left\{\begin{array}{lll}
j_{a} & q & j_{b} \\
J_{b} & l & J_{a}
\end{array}\right\}\left\{\begin{array}{lll}
j_{a} & q & j_{b} \\
J_{b} & l^{\prime} & J_{a}
\end{array}\right\} \\
& \times\left(\delta_{l l^{\prime}}-S_{v_{b} j_{b} l^{\prime}, v_{b} j_{b}}^{J_{b}} S_{v_{a} j_{a} l^{\prime}, v_{a} j_{a} l}^{* J_{a}}\right) .
\end{aligned}
$$

The cross-section thermal averages, Eqs. (14) and (15), of the form $\left\langle f\left(x, x^{\prime}\right) \sigma(\ldots, E)\right\rangle_{r}$, are calculated according to

$$
\left\langle f\left(x, x^{\prime}\right) \sigma(\ldots ; E)\right\rangle_{r}=\int_{0}^{\infty} \mathrm{d} x x e^{-x} f\left(x, x^{\prime}\right) \sigma(\ldots ; E),
$$

where $x=E / k_{B} T$ and $x^{\prime}=E^{\prime} / k_{B} T$.

\section{APPENDIX B: COMPARISON OF POTENTIALS}

The geometry of the Ar-CO system was characterized by three variables: the $\mathrm{C}-\mathrm{O}$ bond length $r_{\mathrm{CO}}$, the distance $R$ from the argon atom to the center of mass of the $\mathrm{CO}$ molecule, and the angle $\theta$ between the $\mathrm{CO}$ molecular axis and the line connecting the argon atom with the center of mass of $\mathrm{CO}$. $\theta=0$ corresponds to the Ar-OC orientation, and $\theta=180^{\circ}$ to the $\mathrm{Ar}-\mathrm{CO}$ geometry.

The intermolecular interaction potential energy surface (IPES) was defined as the difference between the total energy of the system and the sum of the monomers' energies. The interaction energies corrected for the basis-set superposition error using the counterpoise (CP) method [69] were evaluated with the $\operatorname{CCSD}(\mathrm{T})$ (coupled-cluster with single-, double-, and perturbative triple-excitation model) code [70] using the MOLPRO program 2012.1 version [71].

In the calculations we employed Dunning's augmented standard aug-cc-pVXZ $(X=T, Q, 5,6)$ basis sets [72,73] (denoted aXZ). These bases were extended with a set of $3 s 3 p 2 d 2 f 1 g 1 h$ midbond functions, denoted 332211, with exponents of $0.9,0.3$, and 0.1 for the $s$ and $p$ functions, 0.6 and 0.2 for the $d$ and $f$ functions, and 0.3 for the $g$ and $h$ functions. The midbond functions were placed in the middle of the van der Waals bond. The CO interatomic distance we kept fixed to the experimental $1.128323 \AA$ value [74].

In order to avoid overlapping of midbond functions with the atomic basis functions of $\mathrm{CO}$ in collinear geometries, the midbonds were placed between the Ar atom and the $\mathrm{CO}$ molecule using elliptic coordinates. Hence, in T-shaped geometries the midbond point lay at a distance of $R / 2$ between the $\mathrm{Ar}$ atom and the center of mass of $\mathrm{CO}$, while in collinear geometries it lay halfway between the $\mathrm{Ar}$ atom and the $\mathrm{C}$ or $\mathrm{O}$ atom. The distance between the midbond functions and the center of mass of $\mathrm{CO}$ (denoted $\mathrm{X})$ is defined as

$$
r_{\text {midbond }}=\frac{b}{\sqrt{1-(e \cos \theta)^{2}}},
$$

where

$$
e=\sqrt{1-\frac{b^{2}}{a^{2}}}
$$

$a$ and $b$ are the semimajor and semiminor axes of the ellipse, respectively,

$$
\begin{gathered}
a=\frac{1}{2}\left(R+\frac{r_{\mathrm{CX}}+r_{\mathrm{OX}}}{2}\right), \\
b=\frac{R}{2},
\end{gathered}
$$

$r_{\mathrm{CX}}$ and $r_{\mathrm{OX}}$ are the distances between the center of mass of the $\mathrm{CO}$ molecule and the carbon and oxygen atoms, respectively, and, obviously, $r_{\mathrm{CX}}+r_{\mathrm{OX}}=r_{\mathrm{CO}}$.

We estimated the correlation part of the total interaction energy in the complete basis set (CBS) limit employing the formula proposed by Halkier et al. [75] for the correlationconsistent Dunning's basis-set series,

$$
E_{X Y}^{\mathrm{CBS}}=\frac{E_{X}^{\mathrm{corr}} X^{3}-E_{Y}^{\mathrm{corr}} Y^{3}}{X^{3}-Y^{3}}
$$

where $X, Y$ are cardinal numbers of the basis sets, and $E_{X}^{\text {corr }}, E_{Y}^{\text {corr }}$ are the calculated correlation parts of the interaction energies.

The estimated total interaction energies (including the uncorrelated Hartree-Fock contribution) in the aTZ/aQZ and aQZ/a5Z basis-set pairs are close to each other and for $R=$ $3.7 \AA$ the values are -103.54 and $-103.10 \mathrm{~cm}^{-1}$, respectively. This means that the result obtained with the a5Z basis set is still underestimated by almost $5 \mathrm{~cm}^{-1}$.

Since the authors of Ref. [51] used the $\operatorname{CCSD}(\mathrm{T})$ F12b/a5Z calculated interaction energies (no CP correction) as the starting point for constructing their semiempirical potential, in our analysis we also consider a basis-set convergence of the $\operatorname{CCSD}(\mathrm{T})-\mathrm{F} 12 \mathrm{~b}$ approximation. The explicitly correlated $\operatorname{CCSD}(\mathrm{T})-\mathrm{F} 12$ method [76-79] is becoming quite popular, mainly because contributions to the calculated molecular correlation energies coming from single and double excitations are well converged with relatively small orbital bases. However, it seems that in the case of weak intermolecular interactions the errors introduced by the $\mathrm{a} / \mathrm{b} /$ * 
TABLE III. Basis-set study for T-shaped geometries of the Ar-CO complex with $r_{\mathrm{CO}}=1.128323 \AA$. The calculated interaction energies are given in $\mathrm{cm}^{-1}$; distances, in $\AA$. mb, 332211 midbonds; CBS, complete-basis-set extrapolation. See text for more details.

\begin{tabular}{|c|c|c|c|c|c|c|c|c|c|c|c|c|c|}
\hline \multirow[b]{2}{*}{$R$} & \multicolumn{9}{|c|}{$\operatorname{CCSD}(\mathrm{T})$} & \multicolumn{3}{|c|}{$\operatorname{CCSD}(\mathrm{T})-\mathrm{F} 12 \mathrm{~b}^{\mathrm{a}}$} & \multirow[b]{2}{*}{$\mathrm{Fit}^{\mathrm{b}}$} \\
\hline & aTZ & $\mathrm{aQZ}$ & a5Z & $\mathrm{aTZ}+\mathrm{mb}$ & $\mathrm{aQZ}+\mathrm{mb}$ & $a 5 Z+m b$ & $a 6 Z+m b$ & CBS(aTZ/aQZ) & CBS(aQZ/a5Z) & aTZ & $\mathrm{aQZ}$ & a5Z & \\
\hline 2.9 & 818.22 & 737.51 & 709.36 & 716.52 & 702.82 & 694.16 & 689.97 & 687.43 & 689.74 & 680.58 & 683.83 & 680.10 & 658.76 \\
\hline 3.1 & 277.18 & 226.65 & 208.52 & 206.43 & 201.26 & 197.00 & 194.86 & 192.76 & 195.57 & 184.01 & 189.38 & 187.54 & 178.76 \\
\hline 3.3 & 39.10 & 7.82 & -4.12 & -10.05 & -10.76 & -12.74 & -13.77 & -15.23 & -12.96 & -24.29 & -18.80 & -19.62 & -22.31 \\
\hline 3.5 & -54.09 & -73.30 & -81.33 & -88.07 & -86.83 & -87.66 & -88.13 & -88.96 & -87.59 & -97.97 & -92.86 & -92.99 & -93.17 \\
\hline 3.6 & -72.89 & -87.93 & -94.56 & -101.15 & -99.45 & -99.96 & -100.25 & -100.78 & -99.89 & -109.63 & -104.82 & -104.72 & -104.35 \\
\hline 3.7 & -81.27 & -93.05 & -98.54 & -104.81 & -102.85 & -103.14 & -103.30 & -103.54 & -103.10 & -112.12 & -107.69 & -107.42 & -106.77 \\
\hline 3.8 & -83.00 & -92.26 & -96.81 & -102.64 & -100.58 & -100.72 & -100.79 & -100.76 & -100.74 & -108.99 & -104.96 & -104.59 & -103.83 \\
\hline 3.9 & -80.67 & -87.97 & -91.74 & -97.07 & -95.03 & -95.06 & -95.08 & -94.82 & -95.13 & -102.61 & -99.00 & -98.54 & -97.79 \\
\hline 4.1 & -70.14 & -74.75 & -77.35 & -81.54 & -79.79 & -79.72 & -79.68 & -79.15 & -79.88 & -85.89 & -83.11 & -82.50 & -81.96 \\
\hline 4.3 & -57.50 & -60.48 & -62.27 & -65.41 & -64.05 & -63.96 & -63.91 & -63.34 & -64.15 & -68.95 & -66.88 & -66.17 & -65.87 \\
\hline 4.7 & -36.08 & -37.44 & -38.28 & -40.02 & -39.19 & -39.11 & -39.08 & -38.79 & -39.27 & -42.50 & -41.31 & -40.54 & -40.58 \\
\hline 5.1 & -22.22 & -22.92 & -23.32 & -24.26 & -23.77 & -23.73 & -23.71 & -23.60 & -23.83 & -26.27 & -25.31 & -24.70 & -24.86 \\
\hline 5.6 & -12.42 & -12.76 & -12.93 & -13.32 & -13.12 & -13.10 & -13.09 & -13.03 & -13.16 & -15.09 & -14.11 & -13.78 & -13.94 \\
\hline 6.3 & -5.93 & -6.06 & -6.12 & -6.22 & -6.18 & -6.17 & -6.17 & -6.15 & -6.19 & -7.59 & -6.72 & -6.61 & -6.72 \\
\hline 7.0 & -3.08 & -3.13 & -3.15 & -3.17 & -3.17 & -3.17 & -3.17 & -3.17 & -3.17 & -4.04 & -3.45 & -3.47 & -3.53 \\
\hline
\end{tabular}

${ }^{\mathrm{a}} \mathrm{No} \mathrm{CP}$ correction.

${ }^{\mathrm{b}}$ Taken from Ref. [51].

approximations in CCSD-F12 and by a nonexplicitly correlated treatment of perturbative triples (with or without scaling) can become crucial at this level of accuracy [80]. It was also shown that CCSD(T)-F12 methods tend to perform more poorly for larger basis sets.

It is evident from Table III that the interaction energies calculated with the CCSD(T)-F12b method are overestimated in comparison to the estimated CBS limit. They converge very rapidly with the size of the basis set; however, even with the a5Z basis set they are still too large (in absolute value). In Table III we have also added the interaction energies calculated with the fit published in Ref. [51], obtained using their CCSD(T)-F12b/a5Z results. They are slightly different from the a5Z ones and in the vicinity of the lowest-interaction energy geometry the differences are smaller than $1 \mathrm{~cm}^{-1}$. In the short- and long-range distances the curve obtained from the fit lies below the a5Z one, while in the middle range of 3.6-4.3 $\AA$ the former curve runs above the latter.

Calculations of the total potential energy surface (taking into account stretching of the $\mathrm{CO}$ bond) require a compromise between the desired accuracy and the available computational resources. In the following we used the combination of the frozen-core CCSD(T) method and the aug-cc-pV5Z basis set with the 332211 midbond basis set.

To accurately reproduce features of the Ar-CO IPES we decided to carry out calculations of the intermolecular interaction energy for $35 \times 13 \times 8=3640$ geometries of the system. The intermolecular distance $R$ was varied in the range between 1.7 and $20 \AA$ (35 points in total) covering some part of the repulsive wall, the entire van der Waals well, and long-range (interaction energy slightly above $-5 \times$ $10^{-3} \mathrm{~cm}^{-1}$ ) regions. The values of the $\theta$ angle correspond to the abscissas of the 13-point Gauss-Lobatto quadrature. The $r_{\mathrm{CO}}$ intramonomer distance was set to $\left[1.72 a_{0}, 1.86 a_{0}\right.$, $\left.2.00 a_{0}, 2.13992 a_{0}, 2.28 a_{0}, 2.42 a_{0}, 2.56 a_{0}, 2.70 a_{0}\right]$. In order to obtain the values of the IPES for $R$ above $20 \AA$, we fit the calculated points for $R=14.5,17.0$, and $20.0 \AA$ to the following asymptotic function:

$$
\begin{aligned}
V_{\mathrm{as}}(R, r, \theta)= & \frac{C_{6}^{(0)}(r)}{R^{6}} P_{0}(\cos \theta)+\frac{C_{6}^{(2)}(r)}{R^{6}} P_{2}(\cos \theta) \\
& +\frac{C_{7}^{(1)}(r)}{R^{7}} P_{1}(\cos \theta)+\frac{C_{8}^{(0)}(r)}{R^{8}} P_{0}(\cos \theta) \\
& +\frac{C_{8}^{(2)}(r)}{R^{8}} P_{2}(\cos \theta),
\end{aligned}
$$

where $P_{l}(x)$ are the Legendre polynomials and

$$
C_{n}^{(l)}(r)=C_{n}^{(l, 0)}+C_{n}^{(l, 1)} r+C_{n}^{(l, 2)} r^{2} .
$$

The $C_{n}^{(l, p)}$ coefficients are listed in Table IV.

The most apparent difference between our and Sumiyoshi's potential is the difference in their well depths. To illus-

TABLE IV. $C_{n}^{(l, p)}$ coefficients for asymptotic expansion of our IPES, Eqs. (B6) and (B7), for $r$ and $R$ expressed in $\AA$ and $V_{\text {as }}(R, r, \theta)$ expressed in $\mathrm{cm}^{-1}$.

\begin{tabular}{lr}
\hline \hline$C_{6}^{(0,0)}$ & $-2.8457 \times 10^{5}$ \\
$C_{6}^{(0,1)}$ & $1.1592 \times 10^{5}$ \\
$C_{6}^{(0,2)}$ & $-1.4895 \times 10^{5}$ \\
$C_{6}^{(2,0)}$ & $-1.2449 \times 10^{5}$ \\
$C_{6}^{(2,1)}$ & $2.8162 \times 10^{5}$ \\
$C_{6}^{(2,2)}$ & $-1.7265 \times 10^{5}$ \\
$C_{7}^{(1,0)}$ & $2.3698 \times 10^{6}$ \\
$C_{7}^{(1,1)}$ & $-3.0459 \times 10^{6}$ \\
$C_{7}^{(1,2)}$ & $1.2726 \times 10^{6}$ \\
$C_{8}^{(0,0)}$ & $-3.5077 \times 10^{6}$ \\
$C_{8}^{(2,0)}$ & $-3.8155 \times 10^{6}$ \\
\hline \hline
\end{tabular}




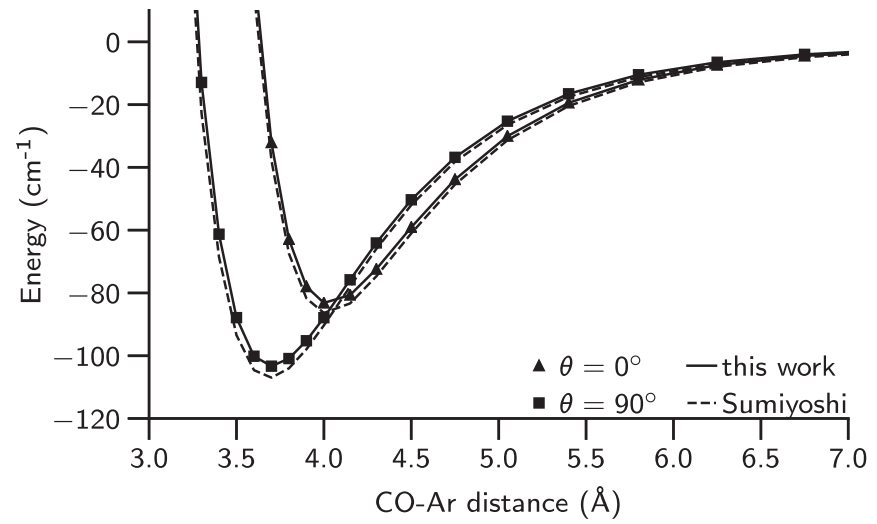

FIG. 3. Our (solid lines) and Sumiyoshi's (dashed lines) potential energy curves for the C-O bond length $r_{\mathrm{CO}}=1.13240 \AA$ and Tshaped (squares) or Ar-OC (triangles) alignment of C-O and Ar. Symbols are shown only on solid lines for clarity.

trate this, we plot the two potentials for $r_{\mathrm{CO}}=1.13240 \AA$ $\left(2.13992 a_{0}\right)$ and two alignments of $\mathrm{CO}$ and Ar: T-shaped (squares) and Ar-OC (triangles) (see Fig. 3). In the Tshaped (Ar-OC) configuration the difference in well depths is $3.709 \mathrm{~cm}^{-1}\left(3.081 \mathrm{~cm}^{-1}\right)$ and the minimum of our potential is displaced from that of Sumiyoshi by $0.0119 \AA(0.0083 \AA)$. There are also notable differences in the short-range part of the potentials, where we expect ours to be more accurate. Figure 4 shows the short-range part of the Legendre expansion of both potentials, averaged over the $\mathrm{CO}$ ground rovibrational state. The short-range part has little effect on the presented lineshape calculations, since our collision energies are limited to $1700 \mathrm{~cm}^{-1}$.

Figure 5 shows the energy dependence of the pressurebroadening and shift cross sections, Eq. (A2), for the $\mathrm{P}(9)$ line for both potentials. For CO-Ar dipole transitions, pressure broadening is predominantly caused by state-changing collisions [81] and the real part of Eq. (A2) can be well approximated by the average total inelastic state-to-state cross

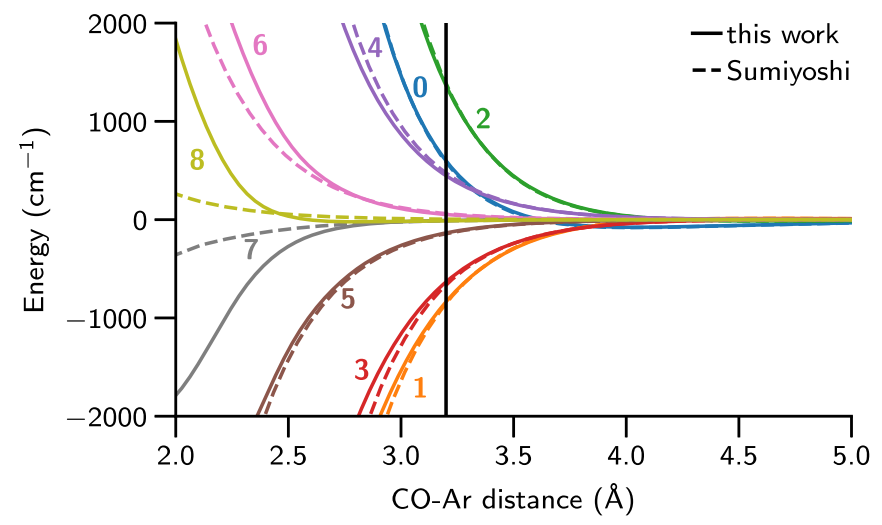

FIG. 4. Comparison between our and Sumiyoshi's rovibrationally averaged Legendre expansions at a small CO-Ar separation. Our potential is represented by solid lines; Sumiyoshi's, by dashed lines. Numbers by the lines label the Legendre orders. The vertical solid black line marks $R=3.2 \AA$, which is the lower $R$ limit of Sumiyoshi's $a b$ initio points.

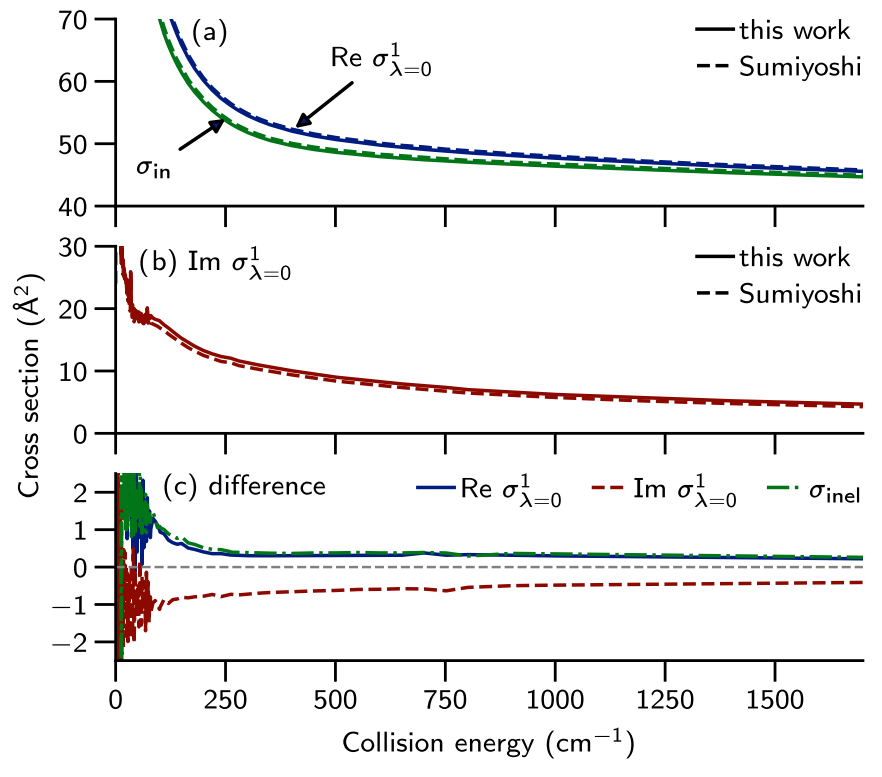

FIG. 5. Spectroscopic cross sections, Eq. (A2), and average total inelastic state-to-state cross sections, Eq. (B8), for the $\mathrm{P}(9)$ line from scattering on both potentials. (a) The broadening cross section (blue) and the inelastic cross section (green); (b) the absolute value of the shift cross section (red); (c) the difference between the values for our potential and that of Sumiyoshi.

section in both radiative levels,

$$
\begin{aligned}
\sigma_{\text {in }}\left(v_{a} j_{a} \rightarrow v_{b} j_{b} ; E\right)= & \frac{1}{2}\left[\sum_{j_{a}^{\prime} \neq j_{a}} \sigma\left(v_{a} j_{a} \rightarrow v_{a} j_{a}^{\prime} ; E\right)\right. \\
& \left.+\sum_{j_{b}^{\prime} \neq j_{b}} \sigma\left(v_{b} j_{b} \rightarrow v_{b} j_{b}^{\prime} ; E\right)\right],
\end{aligned}
$$

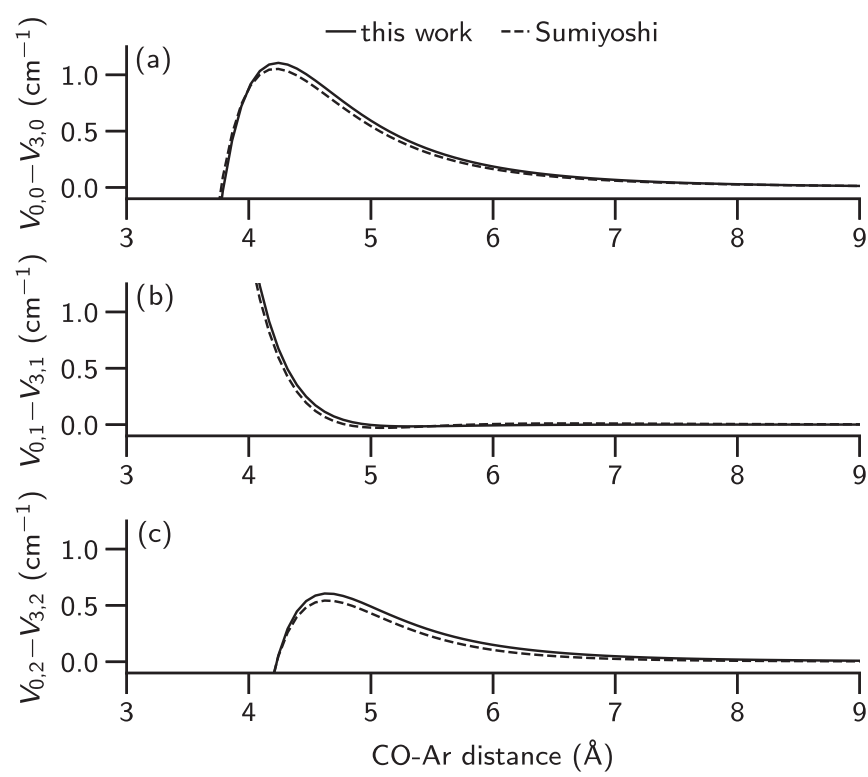

FIG. 6. Differences between Legendre expansion coefficients, $V_{v, l}$, in the lower $(v=0)$ and upper $(v=3)$ vibrational states for both potentials. 
TABLE V. Comparison between pressure-broadening and shift cross sections, Eq. (A2), for Sumiyoshi's (S) and our (HC) potential at $E=262 \mathrm{~cm}^{-1}$. Values for individual potentials are in $\AA^{2}$; the differences $(\mathrm{S}-\mathrm{HC})$ are given as percentages of Sumiyoshi values.

\begin{tabular}{ccccccc}
\hline \hline & Potential & $\mathrm{P}(2)$ & $\mathrm{P}(4)$ & $\mathrm{P}(6)$ & $\mathrm{P}(9)$ & $\mathrm{P}(14)$ \\
\hline & $\mathrm{S}$ & 83.817 & 72.599 & 62.287 & 56.440 & 53.238 \\
$\operatorname{Re} \sigma_{\lambda=0}^{1}$ & $\mathrm{HC}$ & 82.439 & 71.220 & 61.371 & 56.119 & 53.049 \\
& $\mathrm{~S}-\mathrm{HC}$ & 1.6 & 1.9 & 1.5 & 0.6 & 0.4 \\
& $\mathrm{~S}$ & 5.987 & 8.240 & 11.002 & 11.321 & 11.360 \\
$\operatorname{Im} \sigma_{\lambda=0}^{1}$ & $\mathrm{HC}$ & 6.650 & 9.081 & 11.761 & 12.049 & 12.034 \\
& $\mathrm{~S}-\mathrm{HC}$ & -11.1 & -10.2 & -6.9 & -6.4 & -5.9 \\
\hline \hline
\end{tabular}

which is also shown in Fig. 5. Clearly, the difference between broadening cross sections is almost wholly explained by the difference in inelastic cross sections [see Fig. 5(c)]. The shift of a line is determined by elastic collisions only and Sumiyoshi's total elastic collision cross section is the larger of the two potentials, yet the shift is larger for our potential. The more relevant quantity to discuss when comparing line shifts is the difference between the potential energy curves averaged over the upper and the lower vibrational radiative states [27]. In Fig. 6 we present such a comparison for the three lowest Legendre expansion coefficients for both potentials. Our potential consistently shows larger differences, which explains why it produces a larger line shift despite lower elastic collision rate.

In order to confirm that the same trend of larger broadening and smaller shift with the Sumiyoshi potential occurs for other lines, we calculated spectroscopic cross sections, Eq. (A2), for all the other lines at a single kinetic energy, $E=262 \mathrm{~cm}^{-1}$. This energy was selected because it is the most probable energy of $f_{\mathrm{MB}}(E)$ at $T=296 \mathrm{~K}$, therefore the cross sections at this energy can be considered representative of the whole curve. Importantly, this collision energy is also higher than the potential well depth of around $107.1 \mathrm{~cm}^{-1}$, so the cross sections do not probe the Ar-CO resonances that could bias the comparison. This ensures that if $\operatorname{Re} \sigma_{\lambda=0}^{q}\left(\ldots, E=262 \mathrm{~cm}^{-1}\right)$ is larger for one potential relative to the other, then it is safe to assume that the same relation will hold after thermal averaging for $\Gamma_{0}$, and analogously for $\left|\operatorname{Im} \sigma_{\lambda=0}^{q}(\ldots)\right|$ and $\left|\Delta_{0}\right|$. For the $\mathrm{P}(9)$ line, Table $\mathrm{V}$ shows that the differences between the $\operatorname{Re} \sigma_{\lambda=0}^{q}$ and the $\operatorname{Im} \sigma_{\lambda=0}^{q}$ potentials at $E=262 \mathrm{~cm}^{-1}$ closely match the differences for $\Gamma_{0}$ and $\Delta_{0}$ in Table I. Assuming that the comparison is quantitatively accurate also for other lines, we note that the broadening parameters differ the most for the $\mathrm{P}(4)$ line but the agreement between potentials improves for large $j$. This is consistent with the general trend of low $-j$ states being more sensitive to the potential well shape [82-86]. For line shifts, the absolute difference between cross sections stays around $0.7 \AA^{2}$, which improves the relative agreement with increasing $j$ due to the increasing overall value of the shift. We note that a similar weak $j$ dependence of the absolute difference and of the decreasing relative difference is also present in the comparison of experimental and theoretical $\delta_{0}$ values in Table I. This indicates that the vibrational dependence of the potential is an important factor also in explaining the limited accuracy of Sumiyoshi's $a b$ initio $\delta_{0}$ relative to the experimental value.
[1] D. Wunch, G. C. Toon, J.-F. L. Blavier, R. A. Washenfelder, J. Notholt, B. J. Connor, D. W. T. Griffith, V. Sherlock, and P. O. Wennberg, The total carbon column observing network, Philos. Trans. R. Soc. London A 369, 2087 (2011).

[2] C. Frankenberg, R. Pollock, R. A. M. Lee, R. Rosenberg, J.-F. Blavier, D. Crisp, C. W. O’Dell, G. B. Osterman, C. Roehl, P. O. Wennberg, and D. Wunch, The orbiting carbon observatory (OCO-2): Spectrometer performance evaluation using pre-launch direct sun measurements, Atmos. Meas. Tech. 8, 301 (2015).

[3] B. J. Soden, W. D. Collins, and D. R. Feldman, Reducing uncertainties in climate models, Science 361, 326 (2018).

[4] S. Miller, A. Coustenis, P. Read, and J. Tennyson, Characterizing exoplanets, Philos. Trans. R. Soc. London A 372, 20130375 (2014).

[5] I. Gordon, L. Rothman, C. Hill, R. Kochanov, Y. Tan, P. Bernath, M. Birk, V. Boudon, A. Campargue, K. Chance, B. Drouin, J.-M. Flaud, R. Gamache, J. Hodges, D. Jacquemart, V. Perevalov, A. Perrin, K. Shine, M.-A. Smith, J. Tennyson, G. Toon, H. Tran, V. Tyuterev, A. Barbe, A. Császár, V. Devi, T. Furtenbacher, J. Harrison, J.-M. Hartmann, A. Jolly, T. Johnson, T. Karman, I. Kleiner, A. Kyuberis, J. Loos, O. Lyulin, S. Massie, S. Mikhailenko, N. Moazzen-Ahmadi, H. Müller, O. Naumenko, A. Nikitin, O. Polyansky, M. Rey, M. Rotger, S. Sharpe, K. Sung, E. Starikova, S. Tashkun, J. V. Auwera, G. Wagner, J. Wilzewski, P. Wcisło, S. Yu, and E. Zak,
The HITRAN2016 molecular spectroscopic database, J. Quant. Spectrosc. Radiat. Transfer 203, 3 (2017).

[6] P. J. Brewer, J. S. Kim, S. Lee, O. A. Tarasova, J. Viallon, E. Flores, R. I. Wielgosz, T. Shimosaka, S. Assonov, C. E. Allison, A. M. H. van der Veen, B. Hall, A. M. Crotwell, G. C. Rhoderick, J. T. Hodges, J. Mohn, C. Zellweger, H. Moossen, V. Ebert, and D. W. T. Griffith, Advances in reference materials and measurement techniques for greenhouse gas atmospheric observations, Metrologia 56, 034006 (2019).

[7] N. H. Ngo and J.-M. Hartmann, A strategy to complete databases with parameters of refined line shapes and its test for $\mathrm{CO}$ in $\mathrm{He}, \mathrm{Ar}$ and Kr, J. Quant. Spectrosc. Radiat. Transfer 203, 334 (2017).

[8] D. Tran, V. Sironneau, J. Hodges, R. Armante, J. Cuesta, and H. Tran, Prediction of high-order line-shape parameters for air-broadened $\mathrm{O}_{2}$ lines using requantized classical molecular dynamics simulations and comparison with measurements, J. Quant. Spectrosc. Radiat. Transfer 222-223, 108 (2019).

[9] F. Thibault, P. Wcisło, and R. Ciuryło, A test of $\mathrm{H}_{2}-\mathrm{He}$ potential energy surfaces, Eur. Phys. J. D 70, 236 (2016).

[10] L. Gianfrani, Linking the thermodynamic temperature to an optical frequency: Recent advances in Doppler broadening thermometry, Philos. Trans. R. Soc. London A 374, 20150047 (2016).

[11] J. Komasa, K. Piszczatowski, G. Łach, M. Przybytek, B. Jeziorski, and K. Pachucki, Quantum electrodynamics effects in 
rovibrational spectra of molecular hydrogen, J. Chem. Theory Comput. 7, 3105 (2011).

[12] W. Ubachs, J. C. J. Koelemeij, K. S. E. Eikema, and E. J. Salumbides, Physics beyond the standard model from hydrogen spectroscopy, J. Mol. Spectrosc. 320, 1 (2016).

[13] J.-M. Hartmann, H. Tran, R. Armante, C. Boulet, A. Campargue, F. Forget, L. Gianfrani, I. Gordon, S. Guerlet, M. Gustafsson, J. T. Hodges, S. Kassi, D. Lisak, F. Thibault, and G. C. Toon, Recent advances in collisional effects on spectra of molecular gases and their practical consequences, J. Quant. Spectrosc. Radiat. Transfer 213, 178 (2018).

[14] P. R. Berman, Speed-dependent collisional width and shift parameters in spectral profiles, J. Quant. Spectrosc. Radiat. Transfer 12, 1331 (1972).

[15] R. H. Dicke, The effect of collisions upon the Doppler width of spectral lines, Phys. Rev. 89, 472 (1953).

[16] R. Ciuryło, D. A. Shapiro, J. R. Drummond, and A. D. May, Solving the line-shape problem with speed-dependent broadening and shifting and with Dicke narrowing. II. Application, Phys. Rev. A 65, 012502 (2001).

[17] N. H. Ngo, D. Lisak, H. Tran, and J.-M. Hartmann, An isolated line-shape model to go beyond the Voigt profile in spectroscopic databases and radiative transfer codes, J. Quant. Spectrosc. Radiat. Transfer 129, 89 (2013).

[18] P. Wcisło, F. Thibault, H. Cybulski, and R. Ciuryło, Strong competition between velocity-changing and phase- or statechanging collisions in $\mathrm{H}_{2}$ spectra perturbed by Ar, Phys. Rev. A 91, 052505 (2015).

[19] P. Wcisło, F. Thibault, M. Zaborowski, S. Wójtewicz, A. Cygan, G. Kowzan, P. Masłowski, J. Komasa, M. Puchalski, K. Pachucki, R. Ciuryło, and D. Lisak, Accurate deuterium spectroscopy for fundamental studies, J. Quant. Spectrosc. Radiat. Transfer 213, 41 (2018).

[20] R. Wehr, R. Ciuryło, A. Vitcu, F. Thibault, J. R. Drummond, and A. D. May, Dicke-narrowed spectral line shapes of CO in Ar: Experimental results and a revised interpretation, J. Mol. Spectrosc. 235, 54 (2006).

[21] R. Wehr, A. Vitcu, F. Thibault, J. R. Drummond, and A. D. May, Collisional line shifting and broadening in the fundamental Pbranch of CO in Ar between 214 and 324 K, J. Mol. Spectrosc. 235, 69 (2006).

[22] F. Thibault, K. Patkowski, P. S. Żuchowski, H. Jóźwiak, R. Ciuryło, and P. Wcisło, Rovibrational line-shape parameters for $\mathrm{H}_{2}$ in $\mathrm{He}$ and new $\mathrm{H}_{2}-\mathrm{He}$ potential energy surface, J. Quant. Spectrosc. Radiat. Transfer 202, 308 (2017).

[23] G. Kowzan, P. Wcisło, M. Słowiński, P. Masłowski, A. Viel, and F. Thibault, Fully quantum calculations of the line-shape parameters for the Hartmann-Tran profile: A CO-Ar case study, J. Quant. Spectrosc. Radiat. Transfer 243, 106803 (2020).

[24] A. May, W.-K. Liu, F. McCourt, R. Ciuryło, J. Sanchez-Fortún Stoker, D. Shapiro, and R. Wehr, The impact theory of spectral line shapes: A paradigm shift, Can. J. Phys. 91, 879 (2013).

[25] L. Demeio, S. Green, and L. Monchick, Effects of velocity changing collisions on line shapes of HF in Ar, J. Chem. Phys. 102, 9160 (1995).

[26] J.-M. Hartmann, C. Boulet, and D. Robert, Collisional Effects on Molecular Spectra. Laboratory Experiments and Models, Consequences for Applications (Elsevier, Amsterdam, 2008).

[27] C. Luo, R. Wehr, J. R. Drummond, A. D. May, F. Thibault, J. Boissoles, J. M. Launay, C. Boulet, J.-P. Bouanich, and J.-M.
Hartmann, Shifting and broadening in the fundamental band of CO highly diluted in He and Ar: A comparison with theory, J. Chem. Phys. 115, 2198 (2001).

[28] R. Wehr, A. Vitcu, R. Ciuryło, F. Thibault, J. R. Drummond, and A. D. May, Spectral line shape of the $P(2)$ transition in COAr: Uncorrelated ab initio calculation, Phys. Rev. A 66, 062502 (2002).

[29] H. Jóźwiak, F. Thibault, N. Stolarczyk, and P. Wcisło, Ab initio line-shape calculations for the $\mathrm{S}$ and $\mathrm{O}$ branches of $\mathrm{H}_{2}$ perturbed by He, J. Quant. Spectrosc. Radiat. Transfer 219, 313 (2018).

[30] R. Z. Martínez, D. Bermejo, F. Thibault, and P. Wcisło, Testing the ab initio quantum-scattering calculations for the $\mathrm{D}_{2}$ He benchmark system with stimulated Raman spectroscopy, J. Raman Spectrosc. 49, 1339 (2018).

[31] M. Słowiński, F. Thibault, Y. Tan, J. Wang, A.-W. Liu, S.-M. Hu, S. Kassi, A. Campargue, M. Konefał, H. Jóźwiak, K. Patkowski, P. Żuchowski, R. Ciuryło, D. Lisak, and P. Wcisło, $\mathrm{H}_{2}$-He collisions: Ab initio theory meets cavity-enhanced spectra, Phys. Rev. A 101, 052705 (2020).

[32] G. Kowzan, K. Stec, M. Zaborowski, S. Wójtewicz, A. Cygan, D. Lisak, P. Masłowski, and R. S. Trawiński, Line positions, pressure broadening and shift coefficients for the second overtone transitions of carbon monoxide in argon, J. Quant. Spectrosc. Radiat. Transfer 191, 46 (2017).

[33] J. Domysławska, S. Wójtewicz, D. Lisak, A. Cygan, F. Ozimek, K. Stec, C. Radzewicz, R. S. Trawiński, and R. Ciuryło, Cavity ring-down spectroscopy of the oxygen B-band with absolute frequency reference to the optical frequency comb, J. Chem. Phys. 136, 024201 (2012).

[34] A. Cygan, D. Lisak, S. Wójtewicz, J. Domysławska, J. T. Hodges, R. S. Trawiński, and R. Ciuryło, High-signal-to-noiseratio laser technique for accurate measurements of spectral line parameters, Phys. Rev. A 85, 022508 (2012).

[35] D. A. Long, A. Cygan, R. D. van Zee, M. Okumura, C. E. Miller, D. Lisak, and J. T. Hodges, Frequency-stabilized cavity ring-down spectroscopy, Chem. Phys. Lett. 536, 1 (2012).

[36] M. Konefał, M. Słowiński, M. Zaborowski, R. Ciuryło, D. Lisak, and P. Wcisło, Analytical-function correction to the Hartmann-Tran profile for more reliable representation of the Dicke-narrowed molecular spectra, J. Quant. Spectrosc. Radiat. Transfer 242, 106784 (2020).

[37] L. Monchick and L. W. Hunter, Diatomic-diatomic molecular collision integrals for pressure broadening and Dicke narrowing: A generalization of Hess's theory, J. Chem. Phys. 85, 713 (1986).

[38] D. A. Coombe, R. F. Snider, and B. C. Sanctuary, Definitions and properties of generalized collision cross sections, J. Chem. Phys. 63, 3015 (1975).

[39] R. F. Snider and B. C. Sanctuary, Generalized Boltzmann equation for molecules with internal states, J. Chem. Phys. 55, 1555 (1971).

[40] D. A. Shapiro, R. Ciuryło, J. R. Drummond, and A. D. May, Solving the line-shape problem with speed-dependent broadening and shifting and with Dicke narrowing. I. Formalism, Phys. Rev. A 65, 012501 (2001).

[41] P. L. Varghese and R. K. Hanson, Collisional narrowing effects on spectral line shapes measured at high resolution, Appl. Opt. 23, 2376 (1984).

[42] A. S. Pine and A. Fried, Self-broadening in the fundamental bands of HF and HCl, J. Mol. Spectrosc. 114, 148 (1985). 
[43] S. G. Rautian and I. I. Sobel'man, The effect of collisions on the Doppler broadening of spectral lines, Sov. Phys. Usp. 9, 701 (1967).

[44] A. S. Pine, Asymmetries and correlations in speed-dependent Dicke-narrowed line shapes of argon-broadened HF, J. Quant. Spectrosc. Radiat. Transfer 62, 397 (1999).

[45] P. Wcislo, H. Tran, S. Kassi, A. Campargue, F. Thibault, and R. Ciurylo, Velocity-changing collisions in pure $\mathrm{H}_{2}$ and $\mathrm{H}_{2}-\mathrm{Ar}$ mixture, J. Chem. Phys. 141, 074301 (2014).

[46] S. Hess, Kinetic theory of spectral line shapes. The transition between Doppler broadening and collisional broadening, Physica 61, 80 (1972).

[47] S. C. M. Luijendijk, On the shape of pressure-broadened absorption lines in the microwave region. I. Deviations from the Lorentzian line shape, J. Phys. B: At. Mol. Phys. 10, 1735 (1977).

[48] H. M. Pickett, Effects of velocity averaging on the shapes of absorption lines, J. Chem. Phys. 73, 6090 (1980).

[49] D. Priem, F. Rohart, J.-M. Colmont, G. Wlodarczak, and J.-P. Bouanich, Lineshape study of the $J=3 \leftarrow 2$ rotational transition of $\mathrm{CO}$ perturbed by $\mathrm{N}_{2}$ and $\mathrm{O}_{2}$, J. Mol. Struct. 517-518, 435 (2000).

[50] H. Tran, N. H. Ngo, and J. M. Hartmann, Efficient computation of some speed-dependent isolated line profiles, J. Quant. Spectrosc. Radiat. Transfer 129, 199 (2013).

[51] Y. Sumiyoshi and Y. Endo, Three-dimensional potential energy surface of Ar-CO, J. Chem. Phys. 142, 024314 (2015).

[52] A. M. Arthurs and A. Dalgarno, The theory of scattering by a rigid rotator, Proc. R. Soc. London A 256, 540 (1960).

[53] M. S. Child, Molecular Collision Theory (Academic Press, New York, 1974).

[54] D. E. Manolopoulos, An improved log-derivative method for inelastic scattering, J. Chem. Phys. 85, 6425 (1986).

[55] M. H. Alexander and D. E. Manolopoulos, A stable linear reference potential algorithm for solution of the quantum closecoupled equations in molecular scattering theory, J. Chem. Phys. 86, 2044 (1987).

[56] J. M. Hutson and S. Green, MOLSCAT computer code, version 14 (1994); distributed by Collaborative Computational Project No. 6 of the Engineering and Physical Sciences Research Council, UK.

[57] J. M. Hutson and C. R. L. Sueur, MOLSCAT: A program for non-reactive quantum scattering calculations on atomic and molecular collisions, Comput. Phys. Commun. 241, 9 (2019).

[58] M. Danos and S. Geschwind, Broadening of microwave absorption lines due to wall collisions, Phys. Rev. 91, 1159 (1953).

[59] J. Olivero and R. Longbothum, Empirical fits to the Voigt line width: A brief review, J. Quant. Spectrosc. Radiat. Transfer 17, 233 (1977).

[60] J. Tennyson, P. F. Bernath, A. Campargue, A. G. Császár, L. Daumont, R. R. Gamache, J. T. Hodges, D. Lisak, O. V. Naumenko, L. S. Rothman, H. Tran, N. F. Zobov, J. Buldyreva, C. D. Boone, V. M. D. De, L. Gianfrani, J.-M. Hartmann, R. McPheat, D. Weidmann, J. Murray, N. H. Ngo, and O. L. Polyansky, Recommended isolated-line profile for representing high-resolution spectroscopic transitions (IUPAC Technical Report), Pure Appl. Chem. 86, 1931 (2014).
[61] Beyond the binary collision impact approximation, asymmetry of the line can be caused by line mixing and by finite-collisiontime effects $[26,87,88]$. In both cases the asymmetry is described by the dispersive part of the Lorentzian profile, which we found in Ref. [32] to be a poor model for the asymmetry found in our spectra.

[62] P. Wcisło and R. Ciuryło, Influence of the interaction potential shape on the Dicke narrowed spectral line profiles affected by speed-dependent collisional broadening and shifting, J. Quant. Spectrosc. Radiat. Transfer 120, 36 (2013).

[63] R. Blackmore, A modified Boltzmann kinetic equation for line shape functions, J. Chem. Phys. 87, 791 (1987).

[64] See Supplemental Material at http://link.aps.org/supplemental/ 10.1103/PhysRevA.102.012821 for spectroscopic cross sections, HTP line-shape parameters, and our Ar-CO potential energy surface.

[65] http://wcss.pl/

[66] G. C. Corey and F. R. McCourt, Dicke narrowing and collisional broadening of spectral lines in dilute molecular gases, J. Chem. Phys. 81, 2318 (1984).

[67] J. Schaefer and L. Monchick, Line broadening of HD immersed in $\mathrm{He}$ and $\mathrm{H}_{2}$ gas, Astron. Astrophys. 265, 859 (1992).

[68] A. P. Yutsis, I. B. Levinson, and V. V. Vanagas, Mathematical Apparatus of the Theory of Angular Momentum (Israel Program for Scientific Translations, Jerusalem, 1962).

[69] S. Boys and F. Bernardi, The calculation of small molecular interactions by the differences of separate total energies. Some procedures with reduced errors, Mol. Phys. 19, 553 (1970).

[70] K. Raghavachari, G. W. Trucks, J. A. Pople, and M. HeadGordon, A fifth-order perturbation comparison of electron correlation theories, Chem. Phys. Lett. 157, 479 (1989).

[71] H.-J. Werner, P. J. Knowles, G. Knizia, F. R. Manby, M. Schütz et al., Molpro, version 2012.1, a package of ab initio programs (2012); available at: http://www.molpro.net.

[72] T. H. Dunning, Gaussian basis sets for use in correlated molecular calculations. I. The atoms boron through neon and hydrogen, J. Chem. Phys. 90, 1007 (1989).

[73] D. E. Woon and T. H. Dunning, Gaussian basis sets for use in correlated molecular calculations. III. The atoms aluminum through argon, J. Chem. Phys. 98, 1358 (1993).

[74] K. P. Huber and G. Herzberg, Molecular Spectra and Molecular Structure. IV. Constants of Diatomic Molecules (van Nostrand Reinhold, New York, 1979); available at: http://webbook.nist. gov.

[75] A. Halkier, T. Helgaker, P. Jørgensen, W. Klopper, H. Koch, J. Olsen, and A. K. Wilson, Basis-set convergence in correlated calculations on $\mathrm{Ne}, \mathrm{N}_{2}$, and $\mathrm{H}_{2} \mathrm{O}$, Chem. Phys. Lett. 286, 243 (1998).

[76] H. Fliegl, W. Klopper, and C. Hättig, Coupled-cluster theory with simplified linear- $r_{12}$ corrections: The $\operatorname{CCSD}(\mathrm{R} 12)$ model, J. Chem. Phys. 122, 084107 (2005).

[77] H. Fliegl, C. Hättig, and W. Klopper, Inclusion of the (T) triples correction into the linear- $r_{12}$ corrected coupledcluster model CCSD(R12), Int. J. Quantum Chem. 106, 2306 (2006).

[78] T. B. Adler, G. Knizia, and H.-J. Werner, A simple and efficient CCSD(T)-F12 approximation, J. Chem. Phys. 127, 221106 (2007).

[79] C. Hättig, D. P. Tew, and A. Köhn, Communications: Accurate and efficient approximations to explicitly correlated coupled- 
cluster singles and doubles, CCSD-F12, J. Chem. Phys. 132, 231102 (2010).

[80] K. Patkowski, On the accuracy of explicitly correlated coupledcluster interaction energies-Have orbital results been beaten yet? J. Chem. Phys. 137, 034103 (2012).

[81] F. Thibault, R. Z. Martinez, J. L. Domenech, D. Bermejo, and J.-P. Bouanich, Raman and infrared linewidths of $\mathrm{CO}$ in $\mathrm{Ar}$, J. Chem. Phys. 117, 2523 (2002).

[82] A. Palma and S. Green, Effect of the potential well on low temperature pressure broadening in CO-He, J. Chem. Phys. 85, 1333 (1986).

[83] C. F. Roche, A. Ernesti, J. M. Hutson, and A. S. Dickinson, An evaluation of existing potential energy surfaces for $\mathrm{CO}_{2}-$ Ar: Pressure broadening and high-resolution spectroscopy of Van der Waals complexes, J. Chem. Phys. 104, 2156 (1996).
[84] C. Roche, A. Dickinson, A. Ernesti, and J. Hutson, Line shape, transport and relaxation properties from intermolecular potential energy surfaces: The test case of $\mathrm{CO}_{2}-\mathrm{Ar}$, J. Chem. Phys. 107, 1824 (1997).

[85] F. Thibault, B. Calil, J. Boissoles, and J. M. Launay, Experimental and theoretical $\mathrm{CO}_{2}-\mathrm{He}$ pressure broadening cross sections, Phys. Chem. Chem. Phys. 2, 5404 (2000).

[86] F. Thibault, B. Calil, J. Buldyreva, M. Chrysos, J.-M. Hartmann, and J.-P. Bouanich, Experimental and theoretical $\mathrm{CO}_{2}-\mathrm{Ar}$ pressure-broadening cross sections and their temperature dependence, Phys. Chem. Chem. Phys. 3, 3924 (2001).

[87] M. Baranger, Problem of overlapping lines in the theory of pressure broadening, Phys. Rev. 111, 494 (1958).

[88] R. Ciuryło and J. Szudy, Line-mixing and collision-time asymmetry of spectral line shapes, Phys. Rev. A 63, 042714 (2001). 